\title{
A new higher-order elastoplastic beam model for reinforced concrete
}

\author{
G. Corre • A. Lebée - K. Sab - M. K. Ferradi · X. Cespedes
}

the date of receipt and acceptance should be inserted later

\begin{abstract}
The present paper introduces a new elastoplastic beam model for reinforced concrete based on a higher-order beam model previously developed [1]. Steel and concrete are both defined as elastoplastic materials. The beam model represents the concrete body whereas rebars are given a specific discretization. A Rankine criterion is used for concrete in both tension and compression, and a closed-form solution for the local projection of the trial stress on the yield surface is formulated. Steel rebars are modelled with $1 \mathrm{D}$ bar elements and added to the global stiffness of the concrete beam model. The kinematics of the higher-order beam model is enriched by a systematic method with displacement modes. This extension of the kinematics leads to local accuracy and yields results comparable to 3D computations. The present reinforced concrete model is validated through a set of case studies. Implemented within the software programs of the company Strains Engineering, the objective is to develop a fast computing and efficient model that can be directly used by engineers.
\end{abstract}

Keywords Plasticity - Higher-order beam models .

Reinforced concrete $\cdot$ Rankine criterion

\section{Introduction}

The company Strains Engineering develops practical and time-efficient numerical tools for engineers. Within the frame-

G. Corre · A. Lebée · K. Sab

Laboratoire Navier, UMR 8205, École des Ponts ParisTech, IFSTTAR, CNRS, Université Paris Est,

77455 Marne-la-Vallée, France

E-mail: arthur.lebee@enpc.fr

E-mail: karam.sab@enpc.fr

G. Corre · M. K. Ferradi · X. Cespedes

Strains Engineering, 23 Avenue d'Italie, 75013 Paris, France

E-mail: mohammed-khalil.ferradi@strains.fr

E-mail: xavier.cespedes@strains.fr work of the development of a software for bridge analysis, Strains Engineering elaborates new beam models for common construction materials such as steel and reinforced concrete and with the support of the Navier laboratory. The reinforced concrete model presented in this paper is part of these developments.

The development of a beam model for reinforced concrete is complex and faces three main issues. The first difficulty arises in the definition of the constitutive behaviour. Many models of various complexity have been proposed to describe the global and local behaviour of concrete whereas theory of plasticity is almost always used for the material definition of steel. The second difficulty lies in the definition of the kinematics and formulation of the beam model. The beam element considered here should account for axial, shear and bending responses and for their interactions. Finally, the mechanical connection between steel and concrete is singular because of the material contrast and needs regularization.

The first important feature is the definition of the concrete constitutive behaviour. Indeed high stresses in concrete lead to the apparitions of cracks in both tension and compression and has an impact on the strain-stress curve. First, we observe softening which is comparable to a negative hardening. Second it causes damage [2]. Three types of concrete models can be distinguished: damage models, plastic models and plastic-damage models. Damage models efficiently capture the stiffness degradation in the structure but they cannot represent the irreversible deformations $[3,4,5]$. By contrast, plastic models cannot capture the effect of microcracks on the Young modulus but they are suitable for the description of the irreversible deformations they cause $[6,7,8]$. The majority of models therefore consider both damage and plasticity. Most of them are developed with isotropic damage law $[9,10]$. Anisotropic damage models have been developed but their numerical application is much more complex [11]. Numerical applicability of concrete models quickly suffers 
from complex constitutive behaviours. Indeed without regularization techniques, the softening phase in the strain-stress response leads to mesh-dependent solutions. Moreover, in case of softening, both damage and plastic models can lead to multiple discontinuous solutions.

The approach adopted in this paper to overcome this difficulty is the theory of plasticity without softening in order to provide a simple and robust concrete model as a first step. It is however important to mention that the present model allows the introduction of more complex constitutive laws. The Rankine yield criterion is chosen for both tension and compression because of its simplicity and its usage in the engineering community $[12,13]$. A new closed-form projection of the trial stress on the yield surface offering fast integrations of the local equilibrium equations is also presented. The aim of the present model is obviously not to provide a representation of the micro-cracks in concrete, but to yield accurate kinematic descriptions of loaded reinforced concrete structures. Assuming that the degradation of the stiffness in compression is not very important and that concrete strength in tension is very low, the use of a damage parameter may be reasonably neglected. Based on the previous remarks on softening, the choice is made not to consider negative hardening. The main limitation of this approach is the infinite energy the beam can dissipate without restriction. Consequently the present model adopts a fracture energy approach in order to limit the energy dissipation in tension. Given the high strength of concrete in compression, the energy dissipation in compression will not be an issue in most cases.

The second originality of this paper is the definition of a kinematically accurate beam model. A most natural way to define an elastoplastic beam model is to define the constitutive law directly in terms of generalized variables. It requires a preliminary analysis for the computation of the linear and non-linear diagrams of stress-resultants: axial and shear forces, bending moment and torque $[14,15,16]$ or even additional kinematic descriptors $[17,18,19,20]$. If several stress-resultants are considered, their linear and non-linear interactions should therefore be also considered. By solving the non-linear problem in terms of 1D generalized stress at each longitudinal integration points, this approach prevents costly local integrations and provides fast computations [21]. Stress-resultant models are frequently used to study the ultimate load of frames [22] but have also been used for other slender structures such as bridges [5]. However the computation of the stress-resultants interactions can turn out to be tough. Bui and al. [23] choose not to explicitly consider the interaction between shear and bending in their beam element but to represent it through the assembly of two elements. The main limitation of these models is their poor local accuracy since they are limited by the beam theory assumptions and use 1D plastic or damage description.
The common way to improve local accuracy is to use multi-fibers beam models. The beam cross-section is discretized in layers for 2D-beams or in fibers for 3D-beams. One of the first mention of multi-fibers model can be found in the book of Owen and Hinton [24]. These models have since widely been used for linear and non-linear analysis of beam structures. Thanks to its cross-sectional discretization, a multi-fibers model is suitable for describing nonhomogeneous structures such as reinforced concrete beam: some fibers are associated with the concrete properties while others are associated with steel properties thus representing longitudinal rebars. Cross-sectional rebars cannot properly be represented with multi-fibers model. However concrete can be given a modified constitutive law considering the effect of confinement. This model is an intermediate solution between the macroscopic approach of a single beam element and the microscopic approach of a 3D finite element solution. Indeed it provides a microscopic description of the cross-sectional behaviour of the structure while benefiting from the simplified kinematics.

The simplest kinematics is the Euler-Bernoulli kinematics [25, 26, 27] where each cross-section remains plane and normal to the deformed longitudinal axis. It provides an efficient and fast-computing model that accounts for axial and bending effects. However the simple assumptions of the Euler-Bernoulli beam model fails to take shear effects into account. This model is therefore not relevant as soon as shear effects are significant.

For this reason, many developments have been made using the Timoshenko beam model that assumes the crosssection is not necessarily normal to the deformed longitudinal axis. Consequently, it introduces a uniform shear strain through the beam cross-section. Mazars et al. used this kinematics to develop a multi-fibers beam element accounting for shear and torsion for two damage models [28]. As a result, the inclusion of shear effects in the fibers kinematics provides far more accurate damage fields and leads to new failure mechanisms. This approach has therefore been used to study reinforced concrete frames for shear failure analysis [23, 22, 29] or cyclic loading [30], and a careful analysis of the numerical implementation was achieved [31, 32]. However the uniform shear strain introduced with Timoshenko kinematics cannot satisfy the free boundary conditions on the cross-section.

More generally, taking properly interaction between bending moments, shear forces, as well as torsion requires the enrichment of the kinematics with warping functions and was suggested for instance in [33, 34, 35]. However, when material non-linearity such as plastic flow or damage is activated, stress redistribution occurs and the initial kinematics may not remain relevant. In this direction, an elaborated cross sectional analysis was suggested by $[36,37,38]$. This 
approach yields remarkably accurate results but is computationally costly.

In order to preserve local accuracy of the element and to get a better description of macroscopic forces, the approach adopted in the present paper is to use a higher-order elastoplastic beam model. First developed for elasticity in [39], it has been extended to eigenstrains [40] before being adapted to the case of elastoplasticity [1]. The kinematics is enriched thanks to a systematic method with displacement modes computed on the 2D cross-section of the structure. These modes bring local accuracy and can easily describe higher-order shear effects as well as bending or torsion. When required, the kinematics may be updated on the fly according to inelastic strains which occurs in the 3D body [1].

The third issue in the definition of a reinforced concrete beam model is the description of the mechanical connection between steel and concrete. In most reinforced concrete beam models, the numerical description of rebars is often dependent on the definition of the beam model like in the multi-fibers models described previously. In the present approach, the higher-order beam model represents only the concrete body. Steel rebars are therefore added as embedded elements and considered as 1D bars following the kinematics of the higher-order beam. They are meshed independently from concrete which affords a wide range of reinforcement layouts.

The organization of the paper is based on the three issues devised previously. Section 2 is dedicated to the definition of the concrete material: constitutive behaviour, yield criterion and local projection algorithm on the yield surface are presented. Section 3 provides a brief description of the beam model. Steel is then similarly defined in Section 4 and the kinematic connection between concrete and steel rebars is presented. A validation procedure of the present model is then carried out in Section 5. The Application to a rectangular beam is achieved in Section 6 and a fracture-based approach defining the domain of validity of the computed strain-stress curves is introduced. We finally look at the influence of the mesh refinement in Section 7.

\section{A closed-form formulation of Rankine criterion for concrete}

This section introduces the Rankine yield criterion and devises a closed-form projection of the trial stress on the yield surface. This projection prevents from iterative algorithms used for return mapping such as the closest point projection algorithm and improves the time-efficiency of the model.

\subsection{The Rankine yield criterion}

Investigating the shape of the space of plastically-admissible states for concrete materials, Kupfer carried out a series of bi-axial tests [41]. This way, he identified the shape of the yield surface in plane stress. While the limit of compression of concrete was identified as a certain value in uni-axial stress, the limit was about $16 \%$ higher in bi-compression, giving the shape of a square rounded in the bi-compression zone. These experimental results are considered as a reference in civil engineering. However, civil engineers are more likely to use a yield criterion were the compression and tension limits of concrete are constant in uni-axial, bi-axial, or tri-axial compression. This assumption brings simplicity in the analysis of the numerical results, particularly for the identification of the inelastic zones. The Rankine criterion is defined in principal stress as:

$-f_{c} \leq \Sigma_{1}, \Sigma_{2}, \Sigma_{3} \leq f_{t}$

where $f_{c}, f_{t} \geq 0$ are respectively the compression and tension yield limits of the material and $\Sigma_{i}$ for $i=1 . .3$ are the three principal stresses. This criterion is dating from 1876 , and is represented by a cube in principal stress. An isotropic hardening with a very low hardening modulus $H$ is introduced. This choice avoids potential strain localisation problems that can occur with perfect plasticity. Consequently, the usual Rankine criterion defined in Equation (1) is modified as follows:

$-f_{c}-H p \leq \Sigma_{1}, \Sigma_{2}, \Sigma_{3} \leq f_{t}+H p$

where $H$ is the hardening modulus and $p$ is a positive scalar internal variable. The space of plastically-admissible states associated to the criterion defined in Equation (2) is therefore described by the 6 following inequalities:

$f_{1}=\Sigma_{1}-f_{t}-H p \leq 0$,

$f_{2}=\Sigma_{2}-f_{t}-H p \leq 0$,

$f_{3}=\Sigma_{3}-f_{t}-H p \leq 0$

$f_{4}=-\Sigma_{1}-f_{c}-H p \leq 0$,

$f_{5}=-\Sigma_{2}-f_{c}-H p \leq 0$,

$f_{6}=-\Sigma_{3}-f_{c}-H p \leq 0$

The Rankine criterion defined by inequalities of Equation (3) must be considered in the framework of multisurface plasticity. Multisurface plastic criteria are often used when it comes to represent concrete behaviour (see [42, 43]). Considering the stress $\sigma$ and a plastic variable $p$, the space of plastically admissible states $\mathbb{E}_{\sigma}$ is defined in stress-space by:

$\mathbb{E}_{\sigma}=\left\{(\sigma, p) \in \mathbb{S} \times \mathbb{R}_{+} \mid f_{i}(\sigma, p) \leq 0, \forall i \in[1, \ldots, m]\right\}$,

where $\mathbb{S}$ is the space of the 3D stresses. The definition of the functions $f_{i}$ fully characterizes $\mathbb{E}_{\sigma}$. 
The plastic strain $\varepsilon^{\mathrm{p}}$ is computed from the definition of the flow rule. The present flow rule is associative and therefore expresses $\boldsymbol{\varepsilon}^{\mathrm{p}}$ from the yield criterion functions $f_{i}$ as:

$\dot{\boldsymbol{\varepsilon}}^{\mathrm{p}}=\sum_{i=1}^{m} \dot{\gamma}_{i} \frac{\partial f_{i}}{\partial \sigma}$

where $\dot{\gamma}_{i}$ are non-negative scalars called consistency parameters. They must comply with the Kuhn-Tucker complementary conditions:

$\dot{\gamma}_{i} \geq 0, \quad f_{i}(\sigma, p) \leq 0, \quad \dot{\gamma}_{i} f_{i}(\sigma, p)=0$,

and the consistency requirement:

$\dot{\gamma}_{i} \dot{f}_{i}(\sigma, p)=0$

Note that, whereas the limit surface may not be differentiable, each $f_{i}$ may be chosen regular enough. Hence, as $\gamma_{i} \geq 0$, Equation (5) reveals that the plastic flow is in the sub-differential of the limit surface.

\subsection{Closed-form projection on the yield surface}

The method used for the local integration of the constitutive equation is a classic return map algorithm: given a strain increment $\Delta \varepsilon$, an elastic trial stress $\sigma_{n+1}^{\text {trial }}$ is formulated. If $\left(\sigma_{n+1}^{\text {trial }}, p_{n}\right) \in \mathbb{E}_{\sigma}$, the stress is plastically admissible and $\left(\sigma_{n+1}, p_{n+1}\right)=\left(\sigma_{n+1}^{\text {trial }}, p_{n}\right)$. If $\left(\sigma_{n+1}^{\text {trial }}, p_{n}\right) \notin$ $\mathbb{E}_{\sigma},\left(\sigma_{n+1}, p_{n+1}\right)$ is the closest point projection (see [44]) of $\left(\sigma_{n+1}^{\text {trial }}, p_{n}\right)$ onto the boundary $\partial \mathbb{E}_{\sigma}$ in the norm induced by the metric $\boldsymbol{G}$ defined by:

$\boldsymbol{G}=\left(\begin{array}{ll}\boldsymbol{S} & 0 \\ 0 & H\end{array}\right)$,

where $\boldsymbol{S}=\boldsymbol{C}^{-1}$ is the linear elastic compliance matrix of concrete which expression using Voigt notation is given by:

$\boldsymbol{S}=\frac{1}{E}\left(\begin{array}{cccccc}1 & -v & -v & 0 & 0 & 0 \\ -v & 1 & -v & 0 & 0 & 0 \\ -v & -v & 1 & 0 & 0 & 0 \\ 0 & 0 & 0 & 2(1+v) & 0 & 0 \\ 0 & 0 & 0 & 0 & 2(1+v) & 0 \\ 0 & 0 & 0 & 0 & 0 & 2(1+v)\end{array}\right)$

Thus, $\left(\sigma_{n+1}, p_{n+1}\right)$ is the state which satisfies the following minimum principle:

$\left(\sigma_{n+1}, p_{n+1}\right)=\underset{(\sigma, p) \in \mathbb{E}_{\sigma}}{\arg \min }\left\{\frac{1}{2}\left\|\sigma_{n+1}^{\text {trial }}-\sigma\right\|_{S}^{2}+\frac{1}{2} H\left(p_{n}-p\right)^{2}\right\}$

where $\|\sigma\|_{S}=\sqrt{\sigma: S: \sigma}$. The Lagrangian associated with this linearly constrained problem is expressed:

$$
\begin{aligned}
\mathcal{L}\left(\sigma, p, \Delta \gamma_{i}\right)= & \frac{1}{2}\left\|\sigma_{n+1}^{\text {trial }}-\sigma\right\|_{S}^{2}+\frac{1}{2} H\left(p_{n}-p\right)^{2} \\
& +\sum_{i=1}^{6} \Delta \gamma_{i} f_{i}(\sigma, p),
\end{aligned}
$$

and the corresponding Kuhn-Tucker optimality conditions are:

$$
\left.\frac{\partial \mathcal{L}}{\partial \boldsymbol{\sigma}}\right|_{n+1}=\boldsymbol{S}:\left(-\boldsymbol{\sigma}_{n+1}^{\mathrm{trial}}+\boldsymbol{\sigma}_{n+1}\right)+\left.\sum_{i=1}^{6} \Delta \gamma_{i} \frac{\partial f_{i}}{\partial \sigma}\right|_{n+1}=\mathbf{0}
$$

$\left.\frac{1}{H} \frac{\partial \mathcal{L}}{\partial p}\right|_{n+1}=p_{n+1}-p_{n}-\sum_{i=1}^{6} \Delta \gamma_{i}=0$,

$$
f_{i}\left(\sigma_{n+1}, p_{n+1}\right) \leq 0, \quad \Delta \gamma_{i} \geq 0, \quad \Delta \gamma_{i} f_{i}\left(\sigma_{n+1}, p_{n+1}\right)=0 .
$$

Equations (14) are the Kuhn-Tucker complementary conditions. Given that $S$ is isotropic and $f(\sigma, p)$ as well, it can be shown that solving Equation (12) is equivalent to solving its counterpart equation in principal stress, that is:

$\hat{\boldsymbol{S}} \cdot\left(-\boldsymbol{\Sigma}_{n+1}^{\mathrm{trial}}+\boldsymbol{\Sigma}_{n+1}\right)+\left.\sum_{i=1}^{6} \Delta \gamma_{i} \frac{\partial f_{i}}{\partial \boldsymbol{\Sigma}}\right|_{n+1}=\mathbf{0}$

where $\Sigma=\left(\Sigma_{1}, \Sigma_{2}, \Sigma_{3}\right)^{T}$ are the principal stresses expressed as a vector and

$\hat{\boldsymbol{S}}=\frac{1}{E}\left(\begin{array}{ccc}1 & -v & -v \\ -v & 1 & -v \\ -v & -v & 1\end{array}\right)$.

The derivatives of the second term are defined by:

$$
\left(\frac{\partial f_{i}}{\partial \Sigma}\right)_{j}=\frac{\partial f_{i}}{\partial \Sigma_{j}}
$$

The Kuhn-Tucker optimality conditions defined by Equations (13), (14) and (15) are now considered. Assuming that $\left(\Sigma_{n+1}^{\text {trial }}, p_{n}\right) \notin \mathbb{E}_{\sigma}, 26$ situations can be distinguished: $\left(\boldsymbol{\Sigma}_{n+1}, p_{n+1}\right)$ is on one of the 6 plans of the criterion, $\left(\boldsymbol{\Sigma}_{n+1}, p_{n+1}\right)$ is on one of the 12 edges of the criterion or $\left(\boldsymbol{\Sigma}_{n+1}, p_{n+1}\right)$ is on one of the 8 vertices of the criterion.

The 26 local minimum values of $\left(\boldsymbol{\Sigma}_{n+1}, p_{n+1}\right)$ and their associated consistency parameters $\Delta \gamma_{i}$ are given in closed form solutions. The solution of Equation (10) is therefore the minimum of these 26 values. A simple change of basis yields the expression $\sigma_{n+1}$.

The 2D interpretation of the projection of $\left(\Sigma_{n+1}^{\text {trial }}, p_{n}\right)$ onto the yield surface is shown in Figure 1 in the absence of hardening $(H=0)$. Two cases are represented. In the first case, the trial stress is in front of a plane and $\Sigma$ is projected on this plane. In the second case the trial stress is in front of a corner and $\boldsymbol{\Sigma}$ is projected on this corner. The orthogonality represented in Figure 1 must be understood as the norm induced by the metric $\boldsymbol{G}$ defined in Equation (8).

Once the projected state $\left(\sigma_{n+1}, p_{n+1}\right)$ is obtained, the plastic strain increment $\Delta \varepsilon^{\mathrm{p}}$ can be directly computed from 


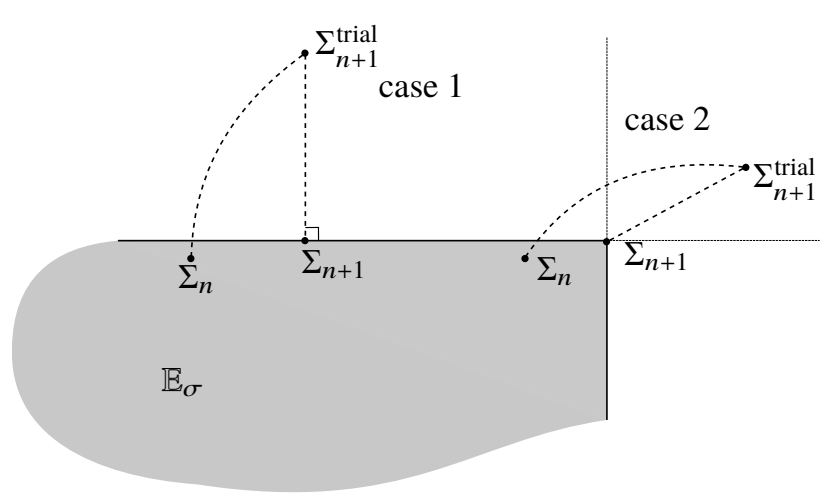

Fig. 1 Orthogonal projection of the trial elastic stress on the yield surface (2D)

the trial stress and its projection. Indeed, the trial stress is expressed:

$\sigma_{n+1}^{\text {trial }}=\sigma_{n}+C: \Delta \varepsilon$

therefore the projected stress $\sigma_{n+1}$ can be written:

$\sigma_{n+1}=\sigma_{n}+C:\left(\Delta \varepsilon-\Delta \varepsilon^{\mathrm{p}}\right)=\sigma_{n+1}^{\mathrm{trial}}-C: \Delta \varepsilon^{\mathrm{p}}$

leading to the expression of the increment of plastic strain:

$\Delta \varepsilon^{\mathrm{p}}=\boldsymbol{S}:\left(\sigma_{n+1}^{\mathrm{trial}}-\sigma_{n+1}\right)$

2.3 Computation of the algorithmic elastoplastic tangent stiffness tensor

We denote by $\mathbb{C}_{\text {act }}$ the array containing the indices of the saturated constraints, that is the constraints for which $f_{i}\left(\sigma_{n+1}, p_{n+1}\right)$ 0 . Then, according to [44], the algorithmic tangent stiffness tensor in the case of multi-surface plasticity is expressed as:

$\boldsymbol{C}_{n+1}^{\mathrm{ep}}=\boldsymbol{D}_{n+1}-\sum_{(i, j) \in \mathbb{C}_{\mathrm{act}}} \frac{\boldsymbol{D}_{n+1}: \partial_{\sigma} f_{i, n+1} \otimes \boldsymbol{D}_{n+1}: \partial_{\sigma} f_{j, n+1}}{\partial_{\sigma} f_{i, n+1}: \boldsymbol{D}_{n+1}: \partial_{\sigma} f_{j, n+1}}$

where $\boldsymbol{D}_{n+1}$ is defined as:

$\boldsymbol{D}_{n+1}=\left[\boldsymbol{S}+\sum_{i \in \mathbb{C}_{\mathrm{act}}} \Delta \gamma_{i} \partial_{\sigma \sigma}^{2} f_{i, n+1}\right]^{-1}$

The derivatives $\partial_{\sigma} f_{i, n+1}$ and $\partial_{\sigma \sigma}^{2} f_{i, n+1}$ are necessary for the computation of the tangent stiffness tensor. The criterion is expressed in the principal stress space and its derivatives according to the general stress are required. The chain rule yields the following expression:

$\frac{\partial f_{i}}{\partial \boldsymbol{\sigma}}=\frac{\partial f_{i}}{\partial \boldsymbol{\Sigma}}: \frac{\partial \boldsymbol{\Sigma}}{\partial \boldsymbol{\sigma}}$
It can be shown that the second derivative has the following expression:

$\frac{\partial^{2} f_{i}}{\partial \sigma^{2}}=\frac{\partial \boldsymbol{\Sigma}}{\partial \boldsymbol{\sigma}}: \frac{\partial^{2} f_{i}}{\partial \boldsymbol{\Sigma}^{2}}: \frac{\partial \boldsymbol{\Sigma}}{\partial \boldsymbol{\sigma}}+\frac{\partial f_{i}}{\partial \boldsymbol{\Sigma}}: \frac{\partial^{2} \boldsymbol{\Sigma}}{\partial \boldsymbol{\sigma}^{2}}$

The first and second derivatives $\partial_{\Sigma} f_{i}$ and $\partial_{\Sigma \Sigma}^{2} f_{i}$ are easy to obtain since the yield surface is analytically expressed in the principal stress. In particular, $\partial_{\Sigma \Sigma}^{2} f_{i}=\mathbf{0}$ for plans, leading to the new relation:

$\frac{\partial^{2} f_{i}}{\partial \sigma^{2}}=\frac{\partial f_{i}}{\partial \boldsymbol{\Sigma}}: \frac{\partial^{2} \boldsymbol{\Sigma}}{\partial \sigma^{2}}$

We denote by $\boldsymbol{n}_{1}, \boldsymbol{n}_{2}$ and $\boldsymbol{n}_{3}$ the three eigenvectors of $\boldsymbol{\sigma}$ corresponding to the 3 eigenvalues $\Sigma_{i}$. The term $\partial_{\sigma} \Sigma$ is then expressed:

$\left(\partial_{\sigma} \boldsymbol{\Sigma}\right)_{l p q r}=\left\{\begin{array}{ll}\left(\boldsymbol{n}_{l} \otimes \boldsymbol{n}_{l}\right)_{q r} & \text { if } l=p \\ 0 & \text { otherwise }\end{array}\right.$,

and for deriving $\partial_{\sigma \sigma}^{2} \Sigma$ we have:

$\frac{\partial \boldsymbol{n}_{l}}{\partial \sigma_{q r}}=\sum_{p=1, p \neq l}^{3} \frac{\boldsymbol{n}_{l} \otimes \boldsymbol{n}_{p}+\boldsymbol{n}_{p} \otimes \boldsymbol{n}_{l}}{\Sigma_{l}-\Sigma_{p}} \cdot \boldsymbol{n}_{l}$

Equation (27) leads to the expression of $\partial_{\sigma \sigma} \Sigma$. The proof of Equation (26) can be found for example in [45]. The derivatives $\partial_{\sigma} f_{i}$ and $\partial_{\sigma \sigma}^{2} f_{i}$ are computed with Equations (25) to (27).

\section{Definition of the beam model}

The concrete body is numerically represented by a higherorder beam model, detailed in this section. The description of steel rebars and their kinematic connection with the concrete body is described in details in Section 4.

\subsection{The AELD beam model}

This paper is based on the elastoplastic beam model developed in [1] called the Asymptotic Expansion Load Decomposition (AELD) beam model. This higher-order elastoplastic beam model does not need any a priori knowledge on the solution of the problem to extend its kinematics.

The displacement modes are computed by using the asymptotic expansion method. This model was first introduced for elastic beams loaded by external forces in [39] and extended to the case of elastic beams loaded by eigenstrains in [40] before its adaptation to elastoplasticity [1].

This higher-order elastoplastic beam model was presented with $J_{2}$-plasticity. Its adaptation to the concrete elastoplastic behaviour presented in Section 2 is straightforward. The main features of this model are briefly recalled. 


\subsection{Description of the beam kinematics}

We consider a beam occupying the prismatic domain $\Omega$. Considering a point $\left(x_{1}, x_{2}, x_{3}\right) \in \Omega$, its $3 \mathrm{D}$ displacement $\boldsymbol{u}$ can be expressed in the following separate form between in-section coordinates and longitudinal coordinates:

$\boldsymbol{u}\left(x_{1}, x_{2}, x_{3}\right)=\sum_{i}^{n} \hat{\boldsymbol{u}}^{i}\left(x_{1}, x_{2}\right) \sum_{j}^{m} N_{j}\left(x_{3}\right) \tilde{u}^{i, j}$

where $\hat{\boldsymbol{u}}^{i}$ are 3D-displacement modes defining the kinematics, $N_{j}$ are the longitudinal interpolation functions and $\tilde{u}^{i, j}$ are the discrete degrees of freedom. The discrete degrees of freedom vector is denoted by $\tilde{\boldsymbol{u}} . n$ and $m$ are respectively the number of displacement modes and the number of interpolation functions.

The displacement modes $\hat{\boldsymbol{u}}^{i}$ are defined on the 2D crosssection of the beam. The displacement modes belong to 2 different categories of modes. Some modes only depend on the geometry of the cross-section. The participation of the loads applied on the structure is captured by other modes, specific to the forces or the eigenstrains considered.

The computation of the modes specific to the geometry and to the forces applied is presented in [39] and the elastoplastic model is presented in [1]. For boundary conditions different from clamped extremities (including linear or surfacic supports) additional modes specific to the boundary conditions are also added in the collection of modes. These modes are used in the elastic and elastoplastic examples presented in Section 5. The boundary conditions are imposed by considering the supports as external forces imposed on the structure and the corresponding new degrees of freedom are introduced. The method is presented in [46].

\subsection{Numerical approximation}

The modes are computed on a transverse mesh of the crosssection $\mathcal{S}$. The discretization used is the same as the one used in [40] and [1]: the modes are computed by using Lagrange quadratic triangle finite elements. Strain and stress are computed and integrated at the 3 Gauss points of each triangle element and the modes of displacement are computed at the nodes of the transverse mesh. NURBS basis functions are used for the longitudinal interpolation. The reasons motivating this choice and a study on NURBS shear locking are presented in [40]. We also define a set of $N_{s}$ longitudinal integration points for the integration of these interpolation functions.

\subsection{Local integration of the equilibrium equations}

The local constitutive equations are integrated over the whole 3D body. A 3D discretization is therefore necessary. This 3D discretization is based on the transverse and longitudinal meshes already defined: the local equations are integrated on a set of cross-sections all transversely discretized with the cross-sectional mesh described previously and placed at the longitudinal positions of the $N_{s}$ longitudinal integration points (Figure 2a). Assuming a generalized displacement increment $\Delta \tilde{\boldsymbol{u}}$, the corresponding 3D strain increment $\Delta \boldsymbol{\varepsilon}$ is computed at the Gauss points of each one of the $N_{s}$ crosssections. This way, the beam is decomposed into sections where the local equations are integrated and the states variables are computed. A natural choice is to place these sections at the positions of the longitudinal integration points of the interpolation functions.

$x_{2}$

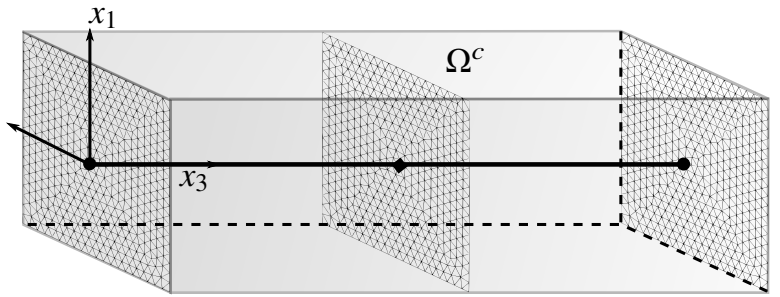

(a) Beam discretization with integration sections representing concrete

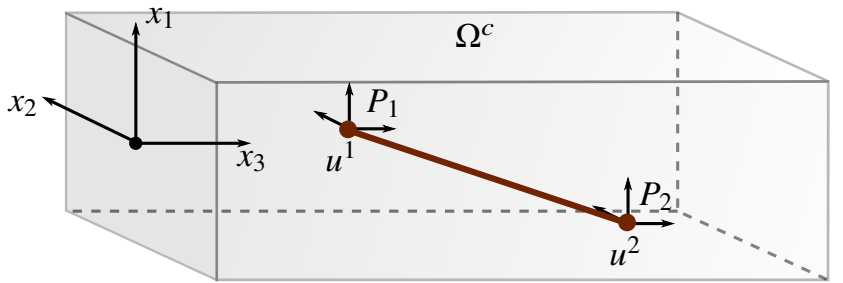

(b) Bar finite element representing the rebar

Fig. 2 Numerical modeling of the concrete body and the steel rebar

\section{Modelling of steel rebars and kinematic connection with concrete}

4.1 Kinematic modelling and elastoplastic behaviour of steel rebars

Steel rebars are often modelled in reinforced concrete models by using homogenization methods. Benefiting from the small cross-sectional dimensions of a rebar compared to the dimensions of the concrete beam and from the larger Young modulus of steel compared to concrete, we can reasonably consider rebars as 1D bar elements.

The young modulus of rebars is $E^{\text {st }}$ and the elastoplastic tangent moduli is denoted by $E^{\mathrm{ep}}$. Steel rebars are considered to be elastoplastic material with an isotropic hardening and 1D Von-Mises criterion. The isotropic hardening modulus of rebars is denoted by $H^{\text {st }}$, and their yield limit $f_{y}^{\text {st }}$. 


\subsection{Kinematic connection of steel and concrete}

Concrete and steel have been assigned an elastoplastic behaviour. We now need to describe the kinematic relation between the two materials. The choice adopted in the present paper is to connect steel to concrete by expressing explicitly the degrees of freedom of rebars in terms of the degrees of freedom of concrete.

The case of a single rebar embedded in a concrete body is first considered. The concrete body is modelled by the beam discretization as represented in Figure 2a, and the rebar is modelled by a bar finite element as represented in Figure $2 b$. The 3D displacement of any point $x \in \Omega^{\mathrm{c}}$ is expressed in Equation (28). As a result, the displacement of the nodes of the steel rebar $P_{1}$ and $P_{2}$ are expressed in terms of the concrete degrees of freedom as follows:

$\boldsymbol{u}^{k}=\sum_{i}^{n} \tilde{\boldsymbol{u}}^{i}\left(x_{\alpha}^{k}\right) \sum_{j}^{m} N_{j}\left(x_{3}^{k}\right) \tilde{u}^{i, j}$,

where $x_{\alpha}^{k}$ and $x_{3}^{k}$ are the positions of the two nodes $(k=1,2)$.

In other words, the steel rebars are driven by the degrees of freedom of concrete and both global tangent stiffness matrice of concrete $\left[\boldsymbol{K}^{\mathrm{ep}, \mathrm{c}}\right]$ and of steel $\left[\boldsymbol{K}^{\mathrm{ep}, \mathrm{st}}\right]$ are expressed according to this kinematics.

This methods would not be efficient if the concrete body were modelled by 3D elements as the rebar would create load concentration on some nodes of the 3D mesh, resulting in displacement singularities. In our beam model, displacements are prescribed by the beam kinematics as expressed by Equation (29). Therefore no singularities occurs unless it is already present in a displacement mode.

This method enables the description of rebars with any orientation in the $3 \mathrm{D}$ global axis system. Therefore, very complex reinforcements can be described by the model. Moreover, since the kinematics adopted for the model is the kinematics of concrete, we avoid unwanted bond-slip issues. It describes correctly the cohesion between rebars and concrete aggregates, assuming that no cracking or debonding occurs.

For the description of reinforcement schemes, rebars are discretized into bar elements. The nodes numerically represent the structural embedding of steel in concrete. The total number of bar elements of rebar $i$ is denoted by $n_{\text {elem }}(i)$ where $i \in\left[1, n_{\text {rebar }}\right]$ and $n_{\text {rebar }}$ is the total number of rebars. The global tangent stiffness matrix can be decomposed on each bar element of each rebar:

$\left[\boldsymbol{K}^{\mathrm{ep}}\right]=\left[\boldsymbol{K}^{\mathrm{ep}, \mathrm{c}}\right]+\sum_{i=1}^{n_{\text {rebar }}} \sum_{j=1}^{n_{\text {elem }}(i)}\left[\boldsymbol{K}^{\mathrm{ep}, \mathrm{st}}\right]^{i, j}$

It is important to note that the discretization of rebars is totally independent from the discretization of the concrete volume. The local integrations of equilibrium equations of concrete and steel can therefore be processed in any order. A mesh sensitivity study is carried out in Section 7.

\section{Validation of the model}

The model has been described in the previous sections with the Rankine criterion. This criterion being not implemented in 3D in classic finite elements softwares (Code_Aster,_Abaqus...), a comparison of a solution using the present model with a reference solution using a different yield criterion would not be relevant. Therefore we suggest to validate and evaluate our model with a step-by-step procedure. First, the model is tested on a case study in elasticity and compared with a reference solution. This first step validates the inclusion of rebars in the model presented in Section 4. Then, the global elastoplastic model is validated by using a Von-Mises criterion for both concrete and steel. This widely used criterion affords an easy comparison with a solution computed with another finite element software. For the final application in the next section, the Von-Mises criterion will be replaced by the Rankine criterion.

\subsection{Reinforced T-beam in elasticity}

In order to validate the integration of rebars in the elastoplastic model, a first case study is conducted in linear elasticity. The results are compared with a 3D reference model computed with the finite element software Code_Aster.

\subsubsection{Description of the case study}

We consider a $10 \mathrm{~m}$-long T-beam simply supported at each end. The section of the T-beam studied is presented in Figure 3. The load is applied over the entire width of the table, between the longitudinal positions $x_{3}=4.5 \mathrm{~m}$ and $x_{3}=5.5 \mathrm{~m}$ as shown in Figure 4.

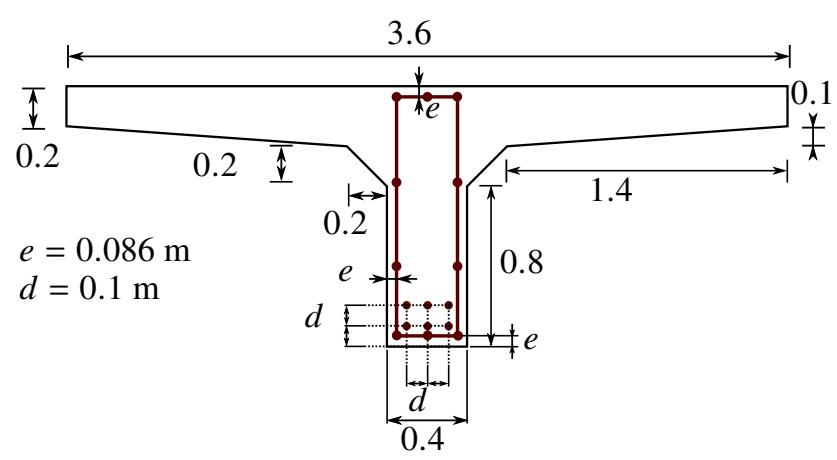

Fig. 3 Geometry of the T-beam section (dimensions in $\mathrm{m}$ ) 


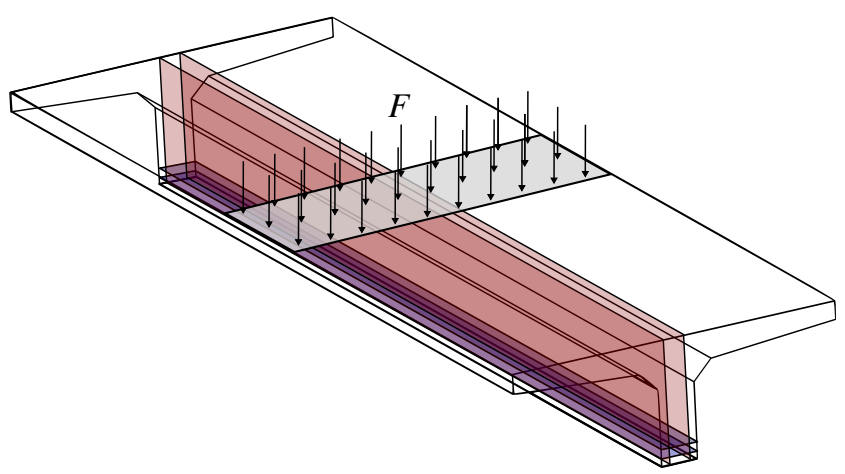

Fig. 4 Surfaces modeling rebars as membranes in the reference solution and description of the load

The beam is reinforced with $2 \times 3$ longitudinal rebars and 11 frames between $x_{3}=0$ and $x_{3}=L$. The boundary conditions and the longitudinal reinforcement are shown in Figure 5. The left support is applied on the entire width of the cross-section and between $x_{3}=0 \mathrm{~m}$ and $x_{3}=0.1 \mathrm{~m}$ where it restrains the three global translations. The right support is applied on the entire width of the cross-section between $x_{3}=9.9 \mathrm{~m}$ and $x_{3}=10 \mathrm{~m}$ where it restrains the transverse translations $\left(u_{\alpha}=0\right)$.

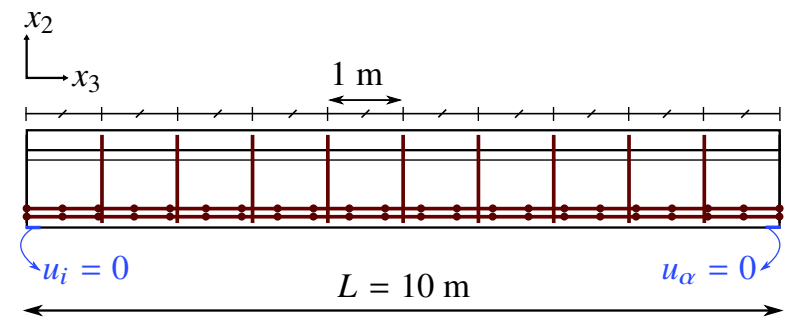

Fig. 5 Boundary conditions and reinforcement of the T-beam

\begin{tabular}{ccc}
\hline & $E$ & $v$ \\
\hline concrete & $20 \mathrm{GPa}$ & 0.2 \\
\hline steel & $200 \mathrm{GPa}$ & - \\
\hline
\end{tabular}

Table 1 Material properties of the linear elastic T-beam

The Young modulus $E$ and the Poisson's ration $v$ of each material are gathered in Table 1. The diameters of longitudinal rebars and frames are respectively $16 \mathrm{~mm}$ and $10 \mathrm{~mm}$. All the distances indicated in Figure 3 are given with respect to the central axis of rebars.

The beam is loaded by a force $F=S \times P$ with $S=3.6 \mathrm{~m}^{2}$ and $P=50 \mathrm{kPa}$ (Figure 4).

\subsubsection{Reference solution}

The reference solution is computed with the finite element software Code_Aster. The concrete is modelled with 3D ele- ments. The elements used here are tetrahedron with quadratic interpolation and 10 Gauss points (Tetra10).

1D bar elements should not be used in 2D and 3D models to represent steel rebars: 1D elements create load concentration on nodes of the 3D mesh and lead to displacement singularities. Consequently, rebars are modelled in the reference solution with anisotropic membrane elements [47] where the strong direction corresponds to the bar direction. Membranes are defined by surfaces based on the 3D mesh and are consequently meshed with triangles. The $2 \mathrm{D}$ interpolation of the triangles is also quadratic (T6 elements).

Longitudinal rebars are modelled with two horizontal membranes represented in blue in Figure 4 and the frames are modelled with two vertical membranes represented in red. Each membrane is characterized by a section per unit length (in $\mathrm{m}^{2} / \mathrm{m}$ ) and by the orientation of rebars.

The mesh characteristics for the reference solution are presented in Table 2.

\begin{tabular}{cccc}
\hline Sol. & concrete & longi. rebars & frames \\
\hline Ref. & 146067 Tetra10 & $2136 \mathrm{~T} 6$ & $8908 \mathrm{~T} 6$ \\
\hline Beam & $477 \mathrm{~T} 6 \times 81$ & 21 bars & 12 bars \\
\hline
\end{tabular}

Table 2 Mesh characteristics for the reference and beam solution

\subsubsection{The beam solution}

Only one cross-sectional mesh is used in the beam model For the present case study, the cross-section of the T-beam is meshed with 477 quadratic triangles (Figure 6).

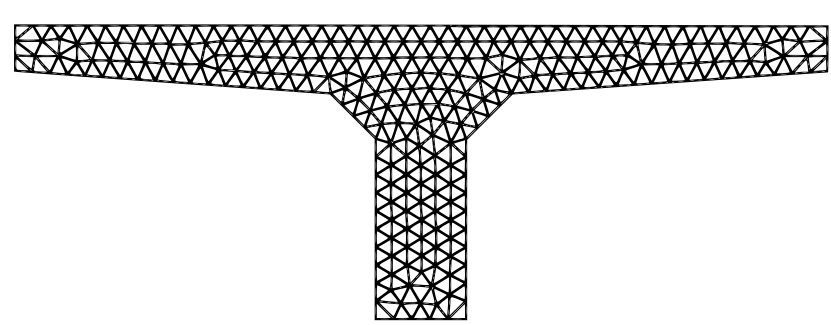

Fig. 6 Cross-sectional mesh of the T-beam for the beam solution

The longitudinal interpolation functions are third-order NURBS and are defined by 21 knots evenly distributed along the longitudinal axis of the beam. The NURBS are integrated with Simpson's method. To ensure an exact integration, 5 integration sections are considered between each knot and the element is therefore locally integrated on 81 integration section also evenly distributed along its axis. The kinematics of the element is composed of 28 displacement modes: 12 specific to the geometry, 6 modes specific to the load applied and 10 modes specific to the boundary conditions. Figure 7 presents the 6 Saint Venant displacement modes as well as 
the displacement modes related to the applied load up to its fifth longitudinal gradient.

The longitudinal transverse frames are respectively discretized with 21 and 12 bars, the corresponding nodes are detailed in Figures 3 and 5.

\subsubsection{Results}

Once the problem solved, the 3D displacement of the beam solution can be easily reconstructed according to Equation 28 . The norm of the 3D displacement computed by the beam solution and the reference solution are compared in Figure 8 (the same scale is adopted for both deformed structures and the magnification factor is 500).

Beyond the consistency of the two solutions, the ability of the beam solution to capture cross-sectional displacements can be here underlined. Indeed, the lowering of the wings of the T-beam is well described by the beam model thanks to its higher-order kinematics.

An interesting feature of the solution adopted for the modelling of rebars in the beam element is that it yields a $3 \mathrm{D}$ viewing of the rebars displacement as shown in Figure 9

In order to better estimate the validity of the beam solution, the displacement and stress of rebars computed from each solution are compared in Figures 10 and 11. Rebars being not individually modelled in the 3D model, we use results computed from the reference solution at the positions corresponding to rebars defined in Figures 3 and 5. Hence in Figure 10, the vertical displacement of two rebars (denominated ST_0 and ST_1) are presented as function of the longitudinal position. The beam solution shows consistent results when compared to the reference solution. The values computed from the beam solution are slightly higher in absolute value: a maximum relative distance of $2.1 \%$ and $2.2 \%$ is respectively observed for ST_0 and ST_1.

The axial stress of ST_0 and ST_1 are presented in Figure 11. The beam solution matches with the reference solution: the relative distance between the two solutions at midspan of the beam $\left(x_{3}=5 \mathrm{~m}\right)$ are respectively $1.7 \%$ and $1.6 \%$ for ST_0 and ST_1. The stress computed from the reference solutions shows oscillations close to the supports. These numerical effects are related to the stiffness contrast between steel and concrete are regularized by the beam model.

Finally, the components of stress in concrete computed from the two solutions at mid-span of the beam $\left(x_{3}=5 \mathrm{~m}\right)$ are compared. Only $\sigma_{\alpha \beta}$ and $\sigma_{33}$ are presented in Figure 12, the shear stresses $\sigma_{\alpha 3}$ vanishing by symmetry.

\subsection{Reinforced T-beam in elastoplasticity}

Section 5.1 has shown the validity of the present reinforced concrete model in elasticity. The model is now studied in elastoplasticity with a Von-Mises criterion for both concrete and steel.

\subsubsection{Description of the case study}

The case study is the same as the one described in Section 5.1.1. Only the material properties are changed and presented in Table 3, where the yield stress $\sigma_{0}$ and the hardening modulus $H$ of each material are also given. A Von-Mises criterion and an isotropic hardening is adopted for both steel and concrete.

\begin{tabular}{ccccc}
\hline & $E$ & $v$ & $\sigma_{0}$ & $H$ \\
\hline concrete & $20 \mathrm{GPa}$ & 0.2 & $10 \mathrm{MPa}$ & $2 \mathrm{GPa}$ \\
\hline steel & $200 \mathrm{GPa}$ & - & $300 \mathrm{MPa}$ & $20 \mathrm{GPa}$ \\
\hline
\end{tabular}

Table 3 Material properties of the elastoplastic T-beam

The beam is loaded by a force $F(t)=S \times P(t)$ with $S=3.6 \mathrm{~m}^{2}$. $P$ takes values between 0 and $700 \mathrm{kPa}$. The load is discretized into 5 increments between 0 and $500 \mathrm{kPa}$ and into 10 increments between $500 \mathrm{kPa}$ and $700 \mathrm{kPa}$.

\subsubsection{Results}

During this case study, the yield stress of concrete and the yield stress of rebars are successively reached. This test provides therefore a good assessment of the accuracy of the elastoplastic beam element presented here.

In order to better evaluate the elastoplastic response of the structure computed from the beam solution, the vertical displacement of rebars ST_0 and ST_1 are presented in Figure 10. The response of both rebars matches very well with the reference solution. A maximum relative distance of $7.510^{-3}$ and $7.110^{-3}$ is respectively observed for ST_0 and ST_1 between both solutions.

The maximum vertical displacement at mid-span of the beam is represented as function of the load applied in Figure 14. This displacement corresponds to the point located at the middle bottom of the section. As shown in the figure, the non-linearity of the elastoplastic behaviour is well captured by the model. The yield stress of concrete is reached after the second increment while the yield stress of steel is reached after the fourth. At the last increment, the beam solution shows a displacement $1.83 \%$ larger than the reference solution. This is consistent with the fact that the beam element appears to be slightly less stiff than the reference solution (see Figures 10 and 13). Differences between results may be explained by the different modelling adopted for rebars in each solution.

The stress in rebar ST_0 at $x_{3}=5 \mathrm{~m}$ is shown in Figure 15 . Three stages are easily identifiable. Both materials are first elastic and the stress progresses linearly in the rebar. 

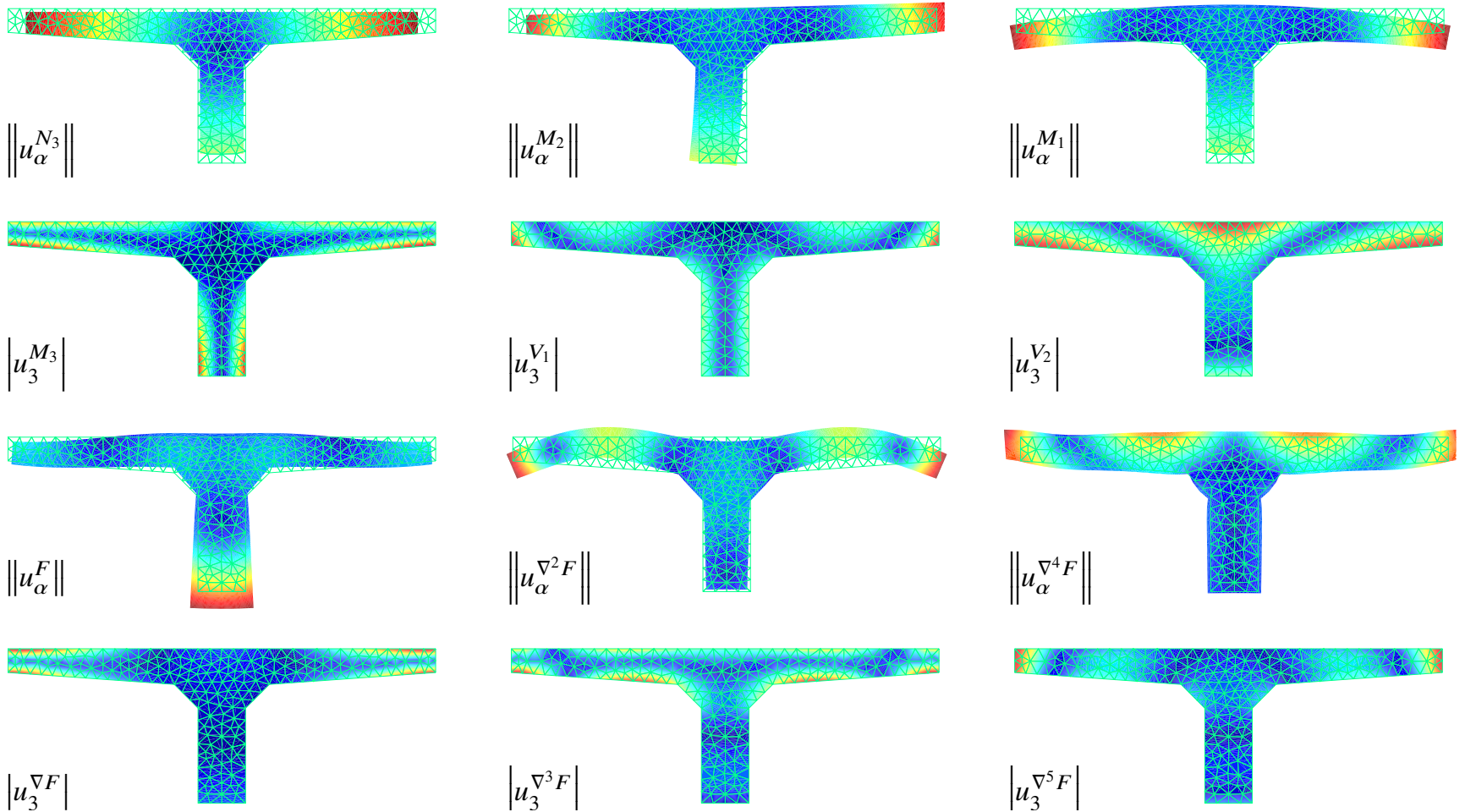

Fig. 7 The 6 Saint Venant displacement modes ( $N_{3}$ longitudinal traction, $M_{\alpha}$ Bending moments, $M_{3}$ torsion warping, $V_{\alpha}$ shear forces warping) and the 6 modes specific to the applied load.

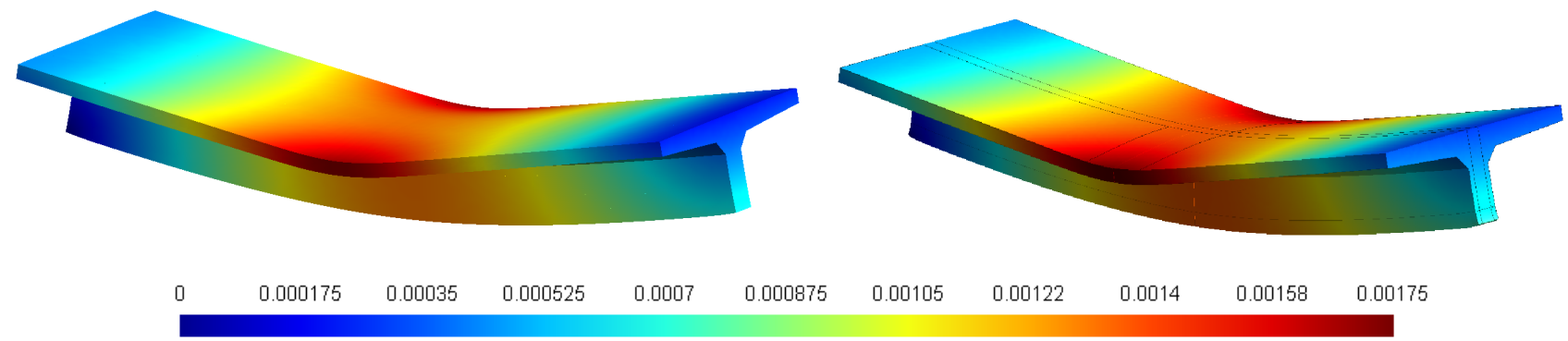

Fig. 8 Deformed shape computed from the beam solution (left) and from the reference solution (right) in elasticity

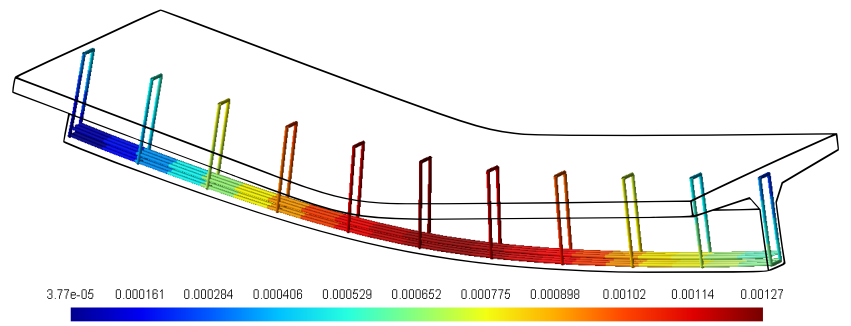

Fig. 9 3D viewing of the displacement of rebars (absolute displacement in meters)

Once concrete has reached plastification, the stress increases faster until the yield stress of steel is reached. The stress then progresses beyond the yield stress according to the plastic modulus. Since the ratio between the Young modulus of steel and concrete is 10 and the concrete yield stress is $10 \mathrm{MPa}$, the concrete yield stress is reached when the stress is $100 \mathrm{MPa}$ in steel. The yield stress of each material is numerically reached between two increments as shown in Figure 15. A finer time discretization should make the three different stages even more distinct.

As long as rebars are elastic, the relative distance between the two solutions is about $10^{-2}$. Once the steel yield stress is reached a relative distance of $2.510^{-2}$ is observed. This gap could be reduced by a finer discretization of rebars (see Section 7).

Finally, the stresses at mid-span of the beam are presented in Figure 16 at the last increment. The normal stress $\sigma_{33}$ is the highest stress in value and the best described by the beam solution when compared to the $3 \mathrm{D}$ model. The axial stress $\sigma_{22}$ is the less satisfactory, but it is consistent with the observation made in elasticity in Figure 12. 


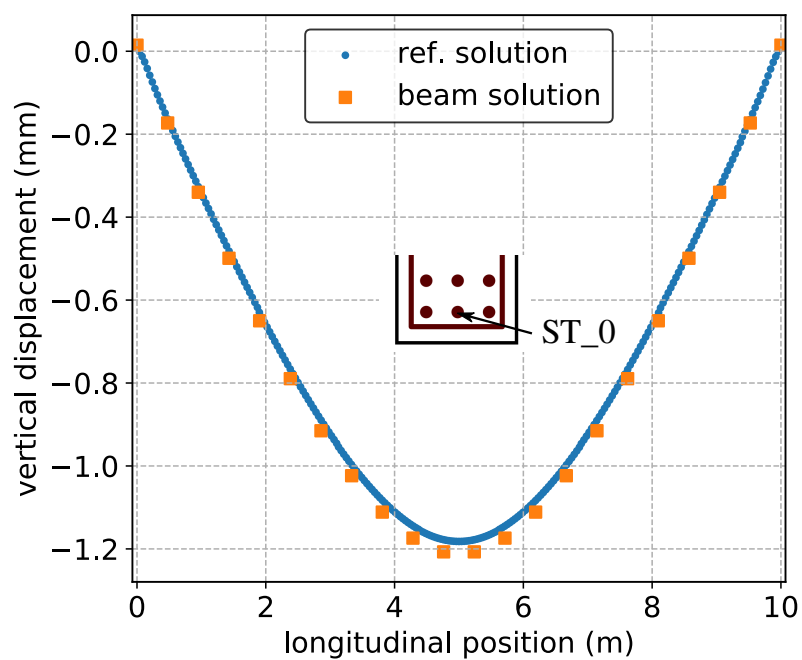

(a) Vertical displacement in rebar ST_0

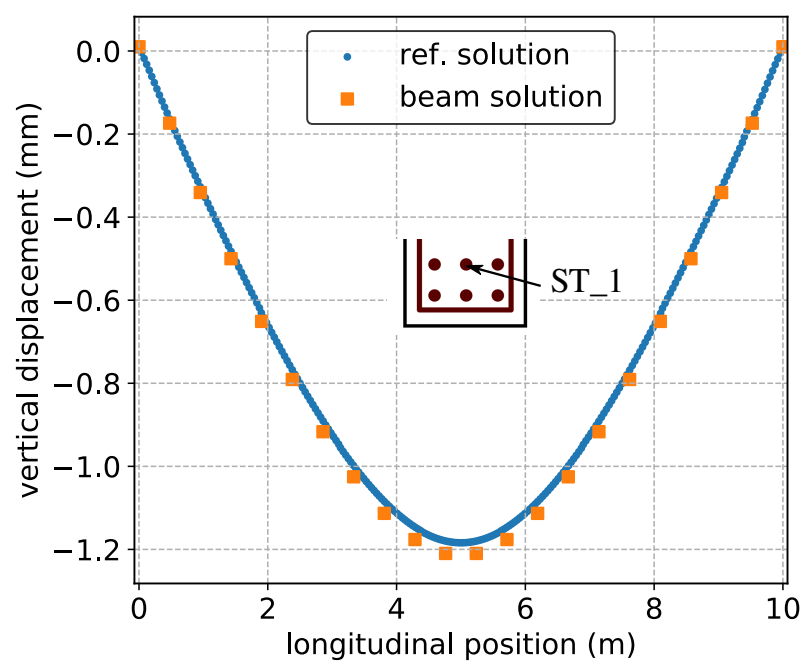

(b) Vertical displacement in rebar ST_1

Fig. 10 Vertical displacement computed from the beam model in rebars ST_0 and ST_1 and from the reference model

\section{Application of the Rankine yield criterion to a rectangular beam}

The numerical behaviour of the present reinforced concrete elastoplastic beam element has been validated in the previous section. The Rankine yield criterion presented in Section 2 is now used instead of the Von-Mises criterion and applied to a doubly clamped rectangular beam.

\subsection{Description of the case study}

We consider a rectangular beam for the present case study: the cross-section of the beam is a rectangle of dimensions $0.4 \mathrm{~m} \times 1.3 \mathrm{~m}$ (Figure 17). The beam is $10 \mathrm{~m}$ long and

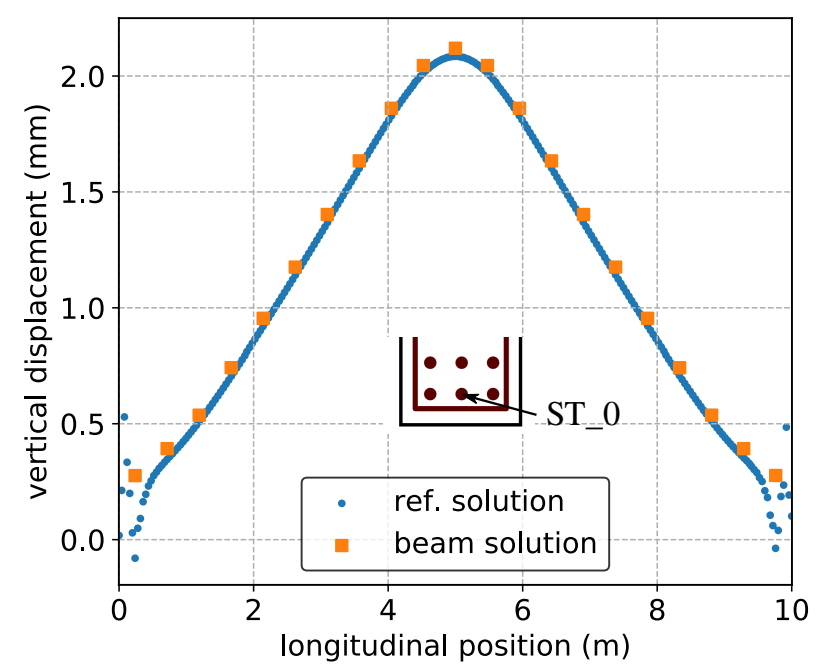

(a) Longitudinal traction in rebar ST_0

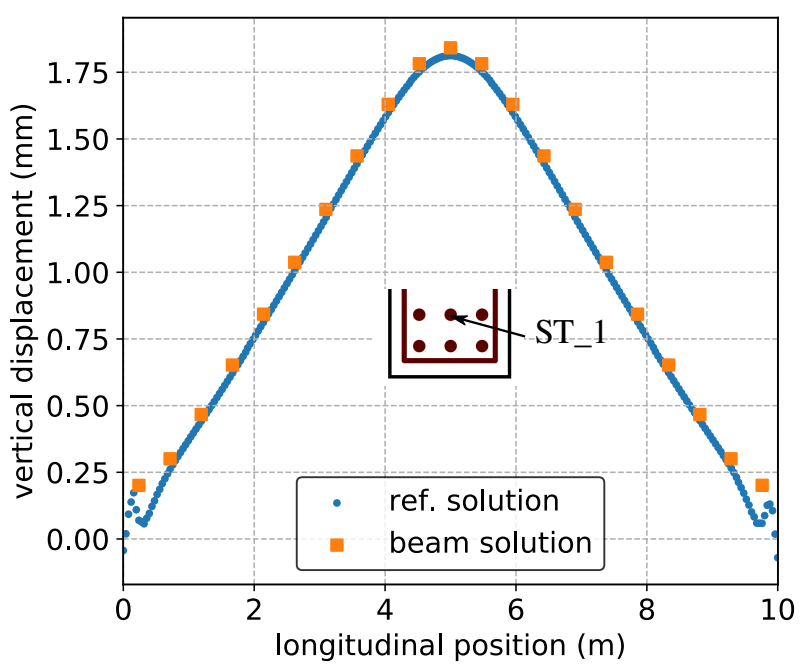

(b) Longitudinal traction in rebar $\mathrm{ST}_{-} 1$

Fig. 11 Longitudinal traction computed from the beam model in rebars ST_0 and ST_1 and from the reference model

clamped at both ends. As a consequence the reinforcement is modified. The cross-sectional reinforcement of the section is as follows : 3 longitudinal rebars are placed at the bottom of the section all along the beam while 3 rebars are placed at the top of the section between $x_{3}=0 \mathrm{~m}$ and $x_{3}=3.5 \mathrm{~m}$ and their symmetric counterparts are placed between $x_{3}=6.5 \mathrm{~m}$ and $x_{3}=10 \mathrm{~m}$. This reinforcement is motivated by the sign shift of the bending moment.

\begin{tabular}{cccc}
\hline & $E$ & $v$ & $H$ \\
\hline Concrete & $20 \mathrm{GPa}$ & 0.2 & $2 \mathrm{~Pa}$ \\
\hline Steel & $200 \mathrm{GPa}$ & - & $2 \mathrm{GPa}$ \\
\hline
\end{tabular}

Table 4 Material properties of the rectangular beam 


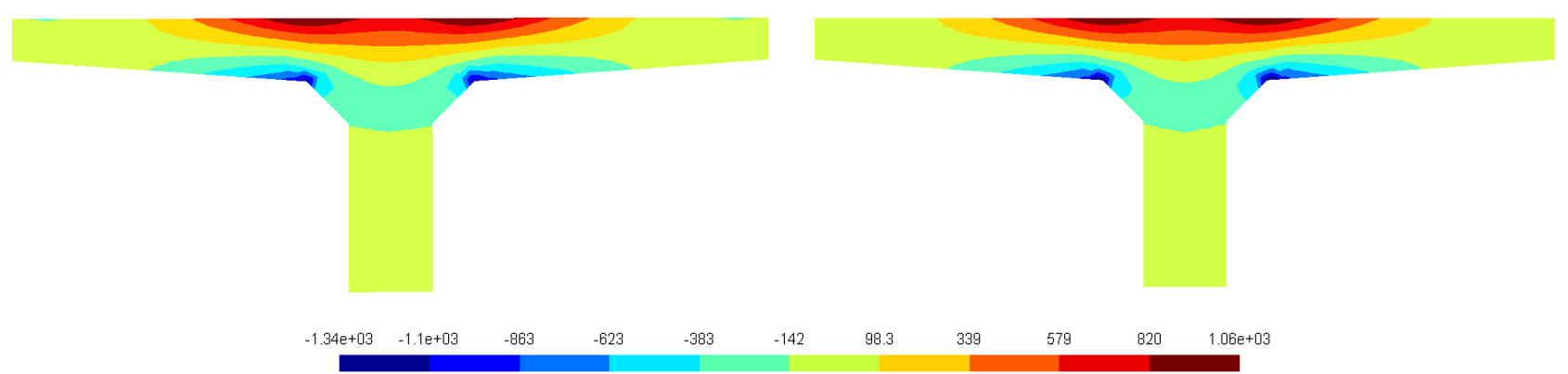

(a) $\sigma_{11}$ computed from the beam solution (left) and from the reference solution (right) in $\mathrm{kPa}$

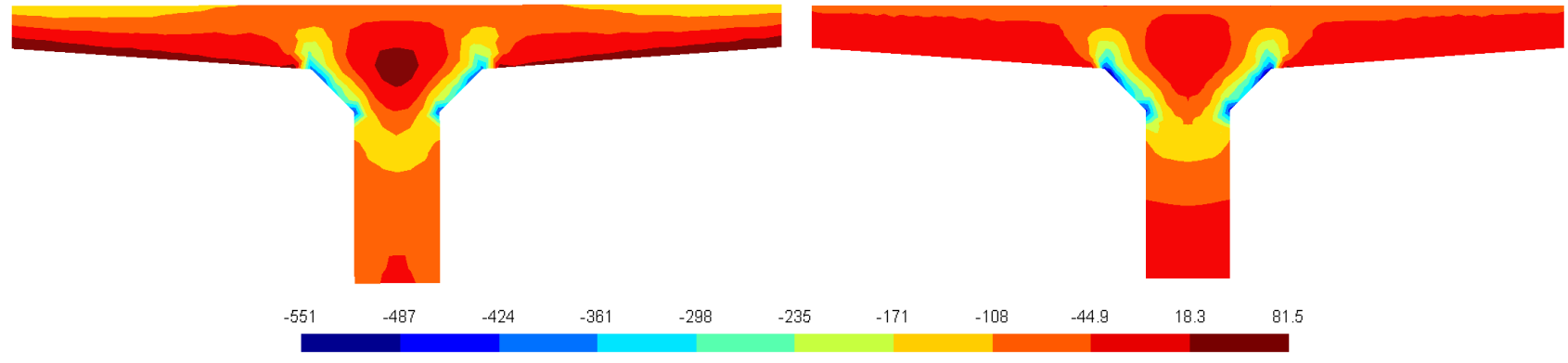

(b) $\sigma_{22}$ computed from the beam solution (left) and from the reference solution (right) in $\mathrm{kPa}$

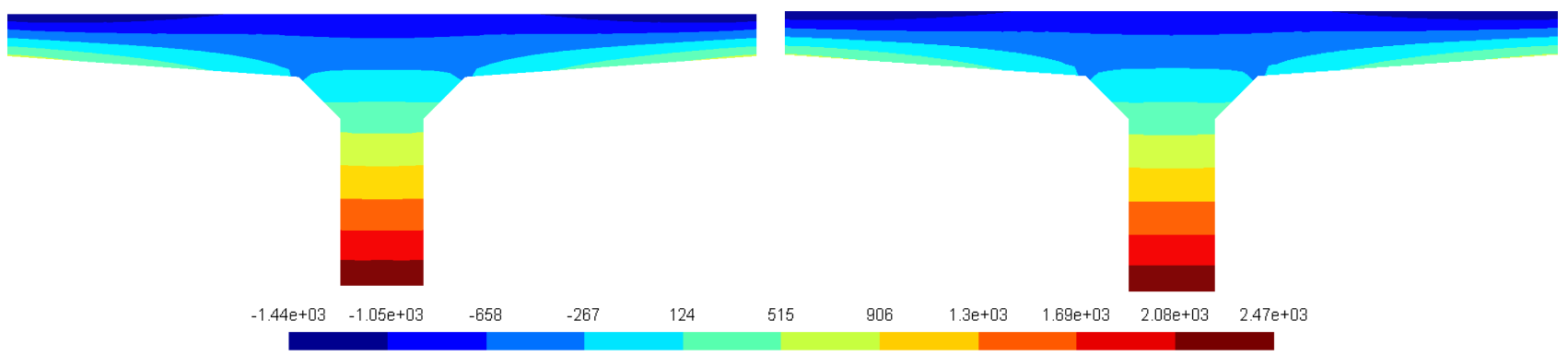

(c) $\sigma_{33}$ computed from the beam solution (left) and from the reference solution (right) in $\mathrm{kPa}$

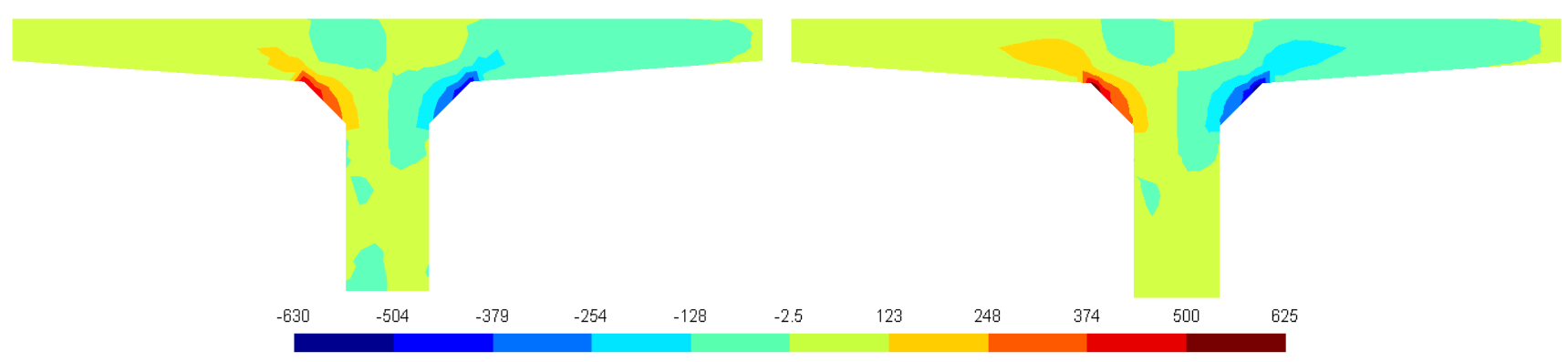

(d) $\sigma_{12}$ computed from the beam solution (left) and from the reference solution (right) in $\mathrm{kPa}$

Fig. 12 Stress computed at mid-span of the beam $\left(x_{3}=5 \mathrm{~m}\right)$ from the beam solution and the reference solution 


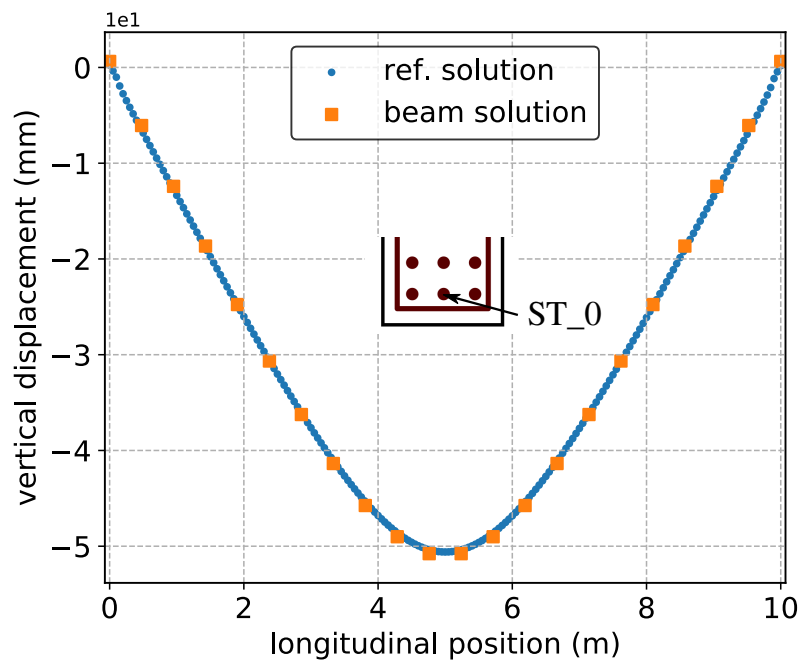

(a) Vertical displacement in rebar ST_0

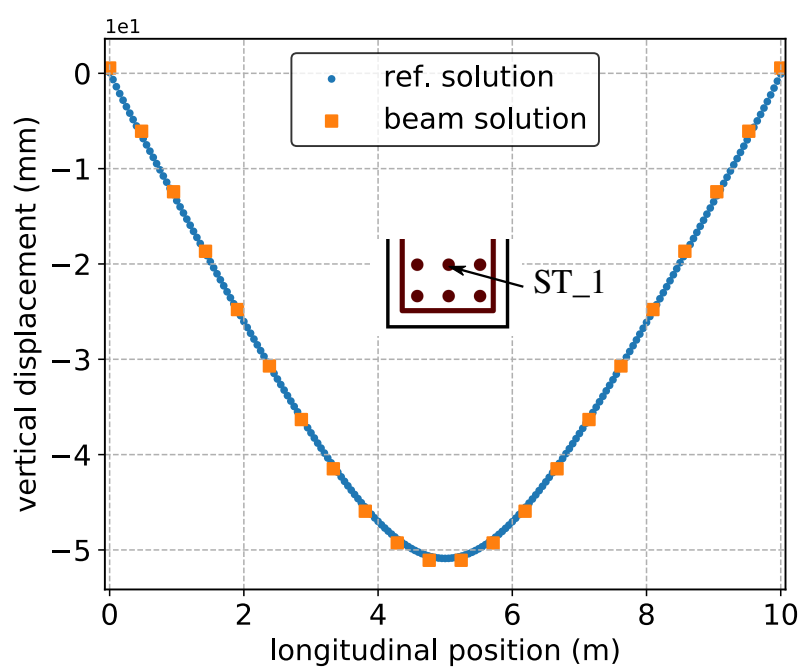

(b) Vertical displacement in rebar ST_1

Fig. 13 Vertical displacement computed from the beam model in rebars ST_0 and ST_1 and from the reference model at the corresponding positions at the last increment

The material properties of the reinforced beam are presented in Table 4. The plastic modulus of concrete is chosen very low since it is only used to avoid potential strain localization problems that can occur in perfect plasticity. The plastic modulus of steel has been chosen lower than actual values in order to emphasize the non-linearity of the global response of the structure. The traction strength of concrete is $f_{t}=2 \mathrm{MPa}$ and its compression yield strength is $f_{c}=40 \mathrm{MPa}$. Rebars still follow a Von-Mises criterion with an isotropic hardening and their yield stress is $300 \mathrm{MPa}$.

The load is decomposed into 30 identical increments of $F_{t}=0.1 \mathrm{MPa}$. The final load is therefore $F_{30}=3 \mathrm{MPa}$.

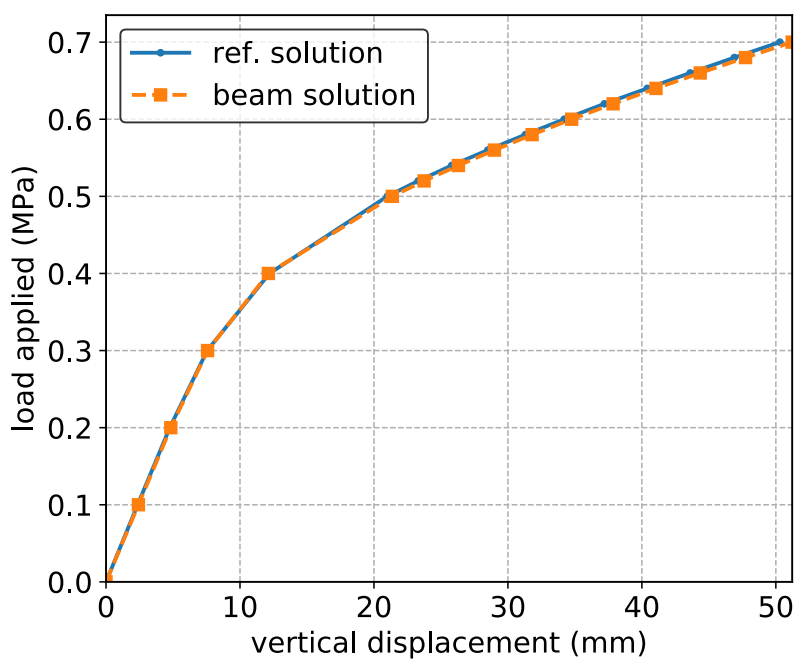

Fig. 14 Maximum vertical displacement at mid-span of the beam

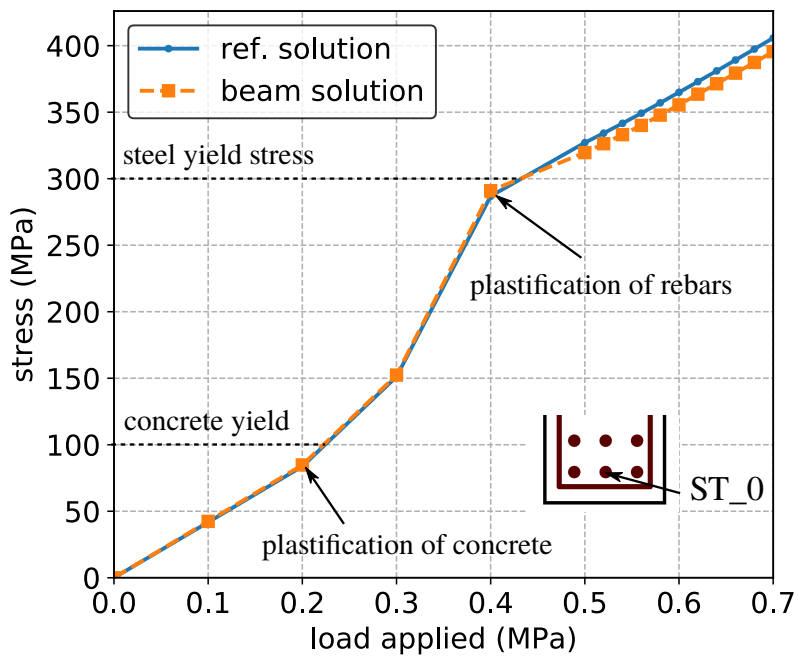

Fig. 15 Stress in ST_0 at mid-span as function of the load applied

\subsection{The beam solution}

The cross-section is meshed with 102 quadratic triangular elements. As for the previous case study, the interpolation functions are third-order NURBS defined by 21 knots evenly distributed along the beam axis. The local integration is processed on 81 integration sections also evenly distributed along the longitudinal axis. The kinematics is composed of 18 displacement modes: 12 modes specific to the geometry and 6 modes specific to the applied load.

\subsection{Results}

The maximum vertical displacement (corresponding to the point located at the middle bottom of the mid-span section) is presented in Figure 18. The successive plastification of 


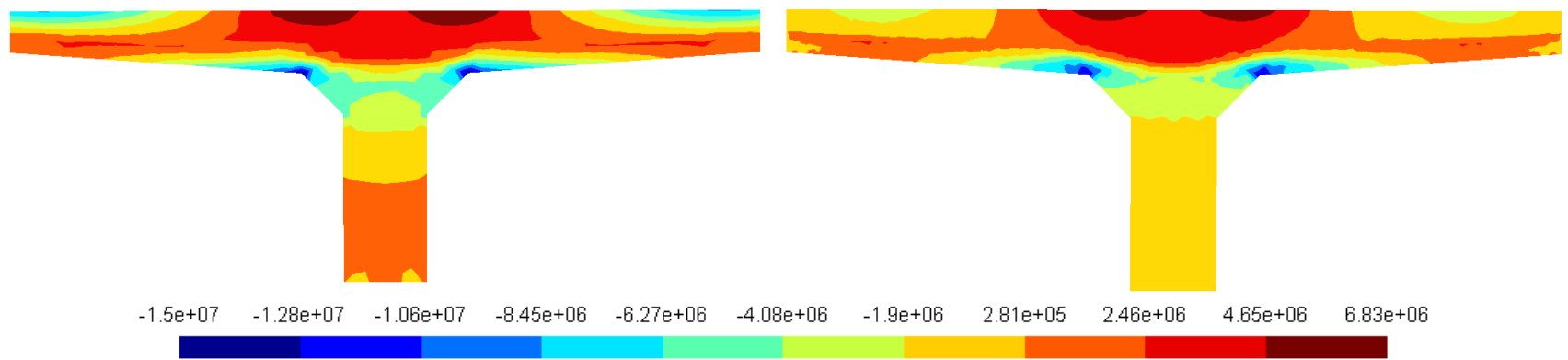

(a) $\sigma_{11}$ computed from the beam solution (left) and from the reference solution (right)

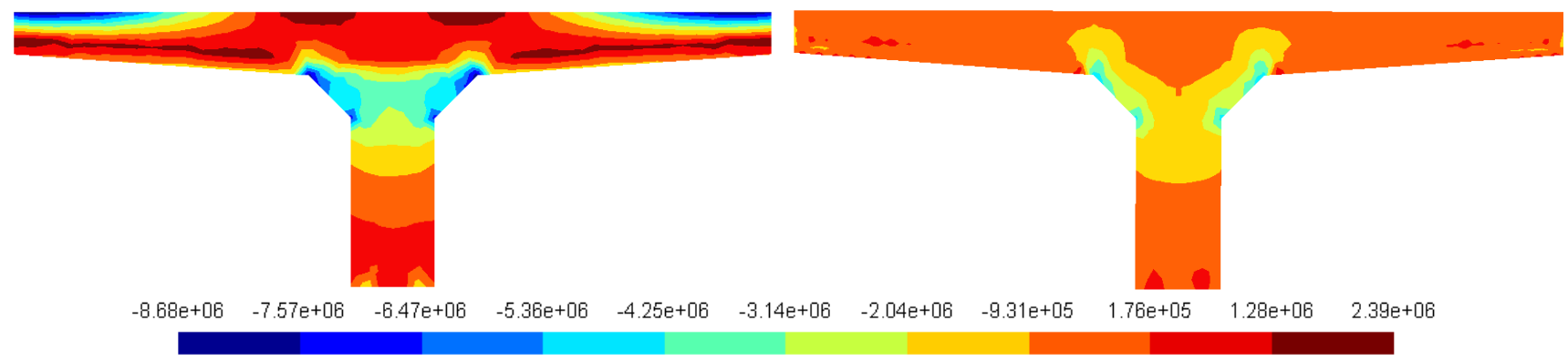

(b) $\sigma_{22}$ computed from the beam solution (left) and from the reference solution (right)

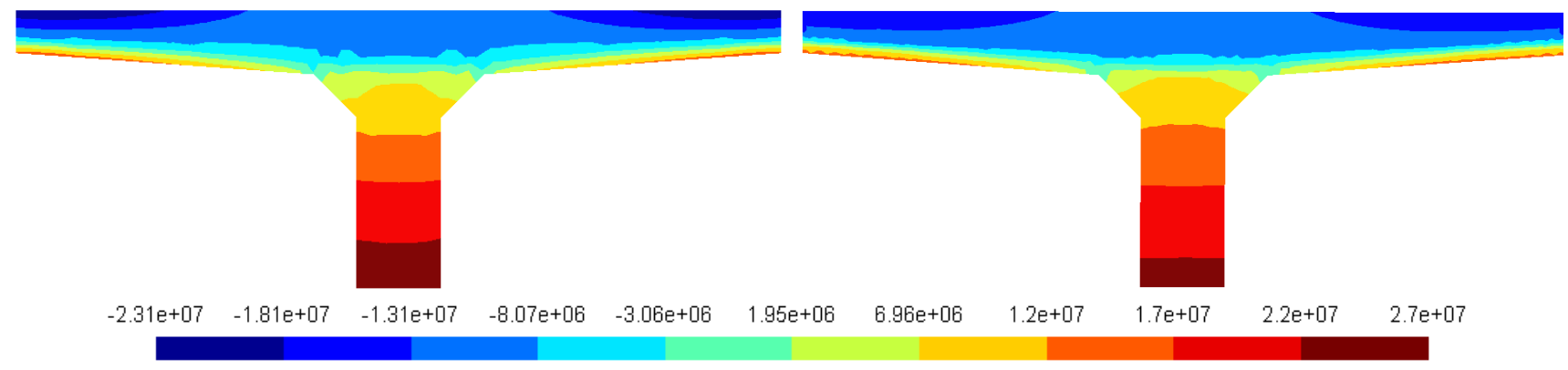

(c) $\sigma_{33}$ computed from the beam solution (left) and from the reference solution (right)

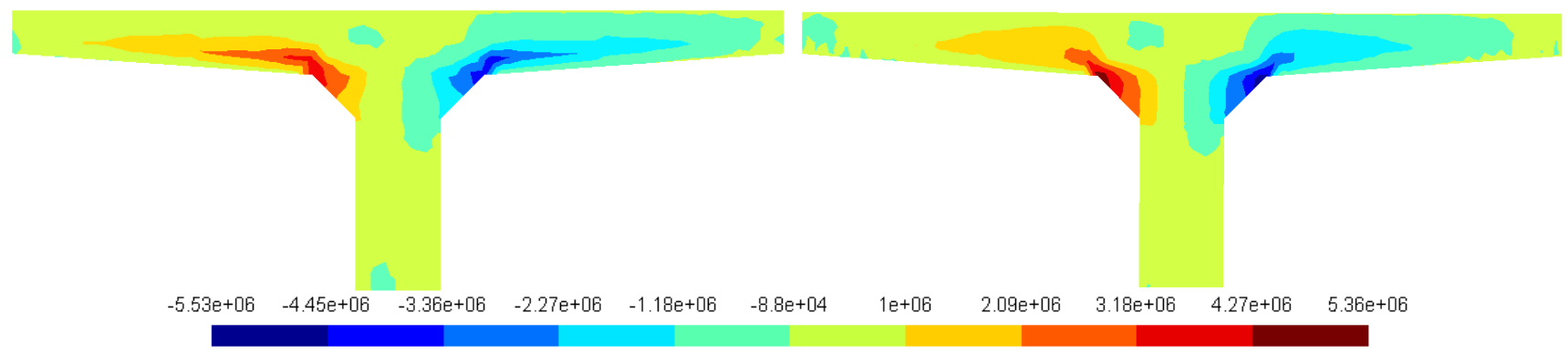

(d) $\sigma_{12}$ computed from the beam solution (left) and from the reference solution (right)

Fig. 16 Stress computed at mid-span of the beam $\left(x_{3}=5 \mathrm{~m}\right)$ from the beam solution and the reference solution 


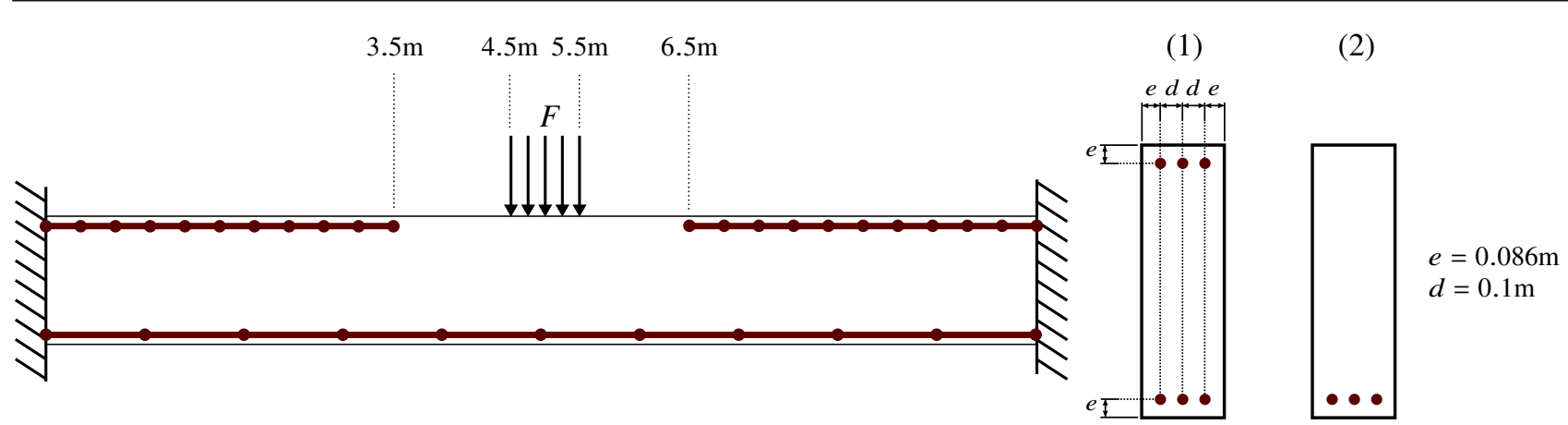

Fig. 17 Longitudinal reinforcement, boundary conditions and cross-sectional reinforcement for $x_{3} \in[0,3.5] \cup[6.5,10](1)$ and $x_{3} \in[3.5,6.5]$ (2)

concrete and steel are identified during the computation and indicated on the figure. Three stages can be distinguished:

1. Concrete and steel are elastic

2. Concrete is plastic and steel is elastic

3. Concrete and steel are plastic

The stage before the plastification of concrete is short but can be identified in the figure. Once concrete has reached $f_{t}$, the loads are transmitted to the clamped end mainly by rebars.

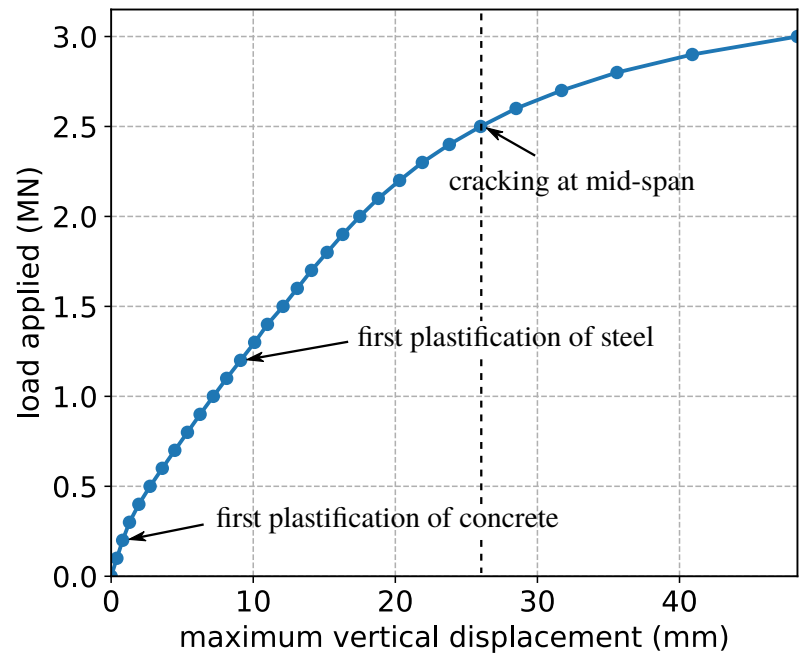

Fig. 18 Maximum vertical displacement computed from the beam model

In Figure 19 is shown the stress computed in rebar ST_3 as function of the load applied on the beam. The stress represented is computed close to the clamped end $\left(x_{3}=0.25 \mathrm{~m}\right)$ where plastification first occurs. The three stages listed above are clearly illustrated. Both materials are elastic until the yield stress of concrete is reached. Since the ratio between the respective Young modulus of steel and concrete is 10 and because the traction yield strength of concrete is $2 \mathrm{MPa}$, stress in steel is $20 \mathrm{MPa}$ when $f_{t}$ is reached in concrete. Once concrete plastifies, the rebar remain elastic but concrete no

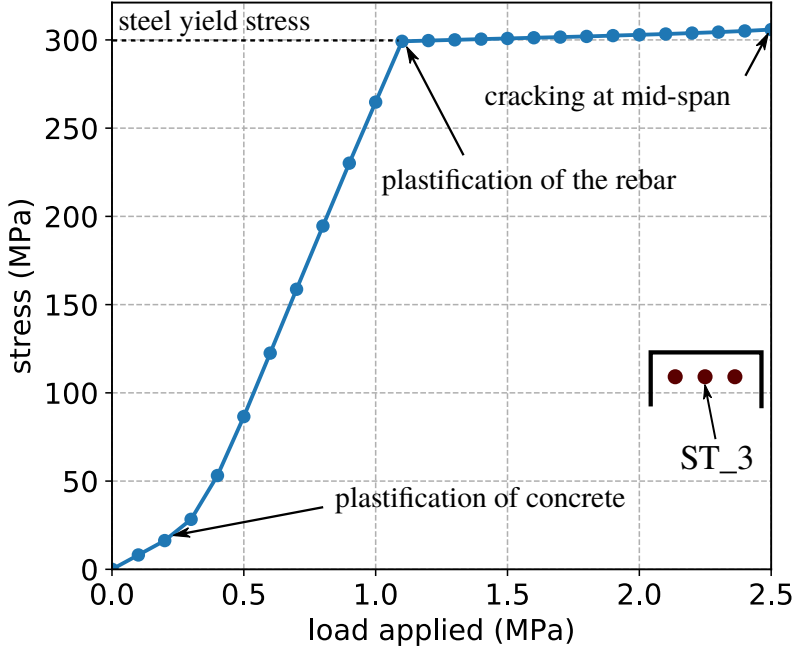

Fig. 19 Stress in rebar ST_3 closed to the clamped extremity $\left(x_{3}=\right.$ $0.25 \mathrm{~m}$ )

longer participates in the load transmission, leading to a new linear stage with a more pronounced increase of the steel stress. Eventually the stress in the rebar reaches the yield stress $(300 \mathrm{MPa})$. The evolution of the stress is then limited due to the low plastic modulus adopted.

Figure 19 shows a satisfactory local response of the model. The global response of the structure to the applied load is illustrated in Figures 20 and 21. Principal stresses of concrete are computed in the whole body and the compression eigenvalue (the lowest eigenvalue) is represented in Figure 20 and the traction eigenvalue (the highest eigenvalue) is represented in Figure 21. Both figures show the same lateral face of the beam at the 6 successive time steps $\left\{t_{5}, t_{10}, t_{15}, t_{20}, t_{25}, t_{30}\right\}$.

The minimum eigenvalues of the 6 time steps take values between $-41 \mathrm{MPa}$ and $1 \mathrm{MPa}$, describing the distribution of compression stresses in the structure. They describe clearly the development of two struts in the structure from the load application to the extremities. The compression limit is first reached at the clamped end at $t_{15}$ and then at the load applica- 
tion area at $t_{20}$. The compression plastified area (in blue) then expands and the compression increases in the two struts. This load transmission was expected and highlights the ability of the present beam model to represent 3D phenomena.

The maximum eigenvalues take values between $-0.2 \mathrm{MPa}$ and 2.2 MPa, describing the distribution of tensile stresses in the concrete body. The traction limit $f_{t}$ is reached at time step $t_{5}$. This is consistent with Figures 18 and 19 where the plastification of concrete seems to occur at $t_{2}$. The three expected areas in traction are distinctly described. These traction eigenvalues are complementary and consistent with compression eigenvalues observed in Figure 20. The empty areas correspond to eigenvalues out of scale.
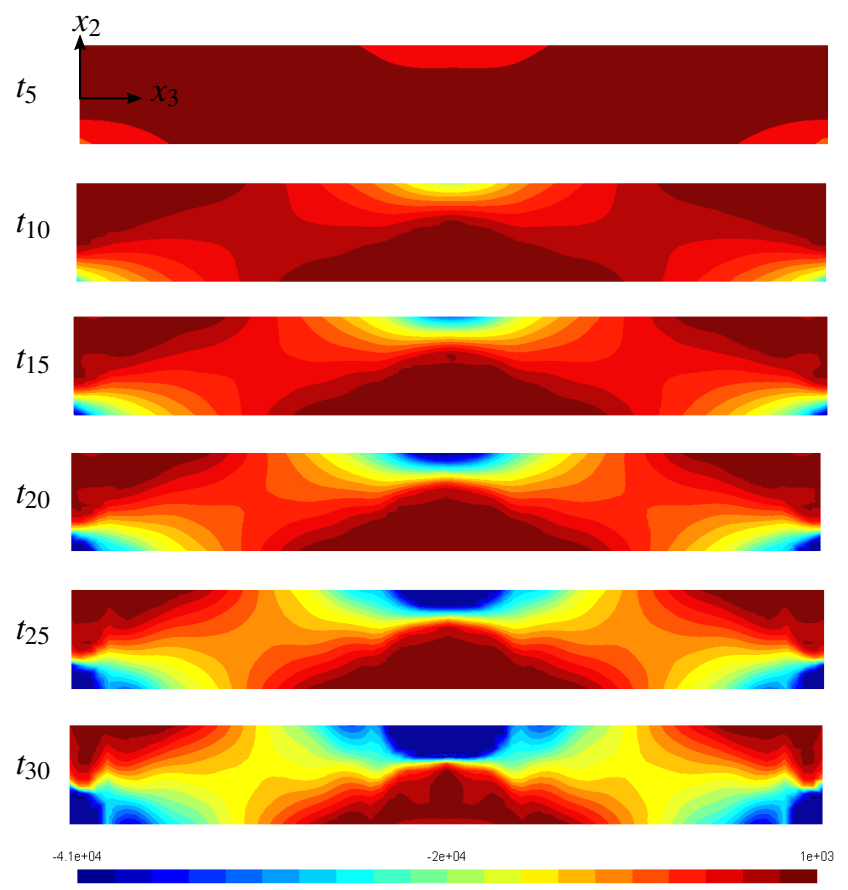

Fig. 20 Evolution of minimum stress eigenvalues in the concrete body (in $\mathrm{kPa}$ )

Finally the normal stress computed at mid-span of the beam $\left(x_{3}=5 \mathrm{~m}\right)$ is presented in Figure 22. The lower half of the section reaches traction limit at time step $t_{5}$. Compression then gradually expands in the upper half until the limit between traction and compression zones reaches at time step $t_{30}$ the vertical coordinate $x_{2}=x_{e}$ such that $\sigma_{33}\left(x_{2}>x_{e}\right)=f_{t}$ and $\sigma_{33}\left(x_{2}<x_{e}\right)=-f_{c}$.

By showing the expected response of the structure, this case study confirms the relevance of the present reinforced concrete elasotplastic beam model. Figures 20 and 21 show that the introduction of the Rankine yield criterion as described in Section 2 leads to a convincing 3D global response of the beam. Moreover, it illustrates the great ability of this beam model to describe $3 \mathrm{D}$ phenomena such as the formation of the struts exhibited here.

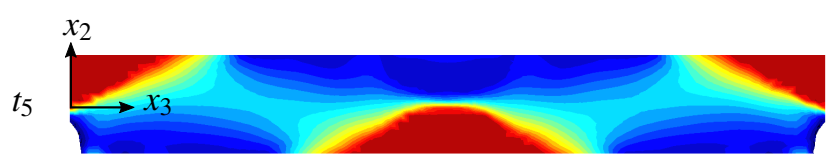

$t_{10}$

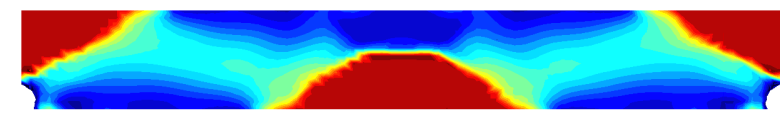

$t_{15}$

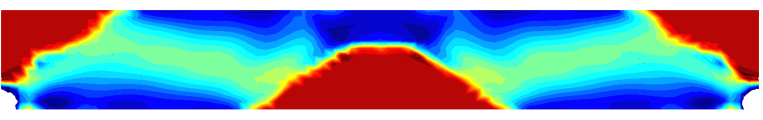

$t_{20}$

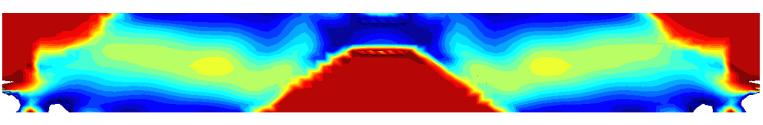

$t_{25}$

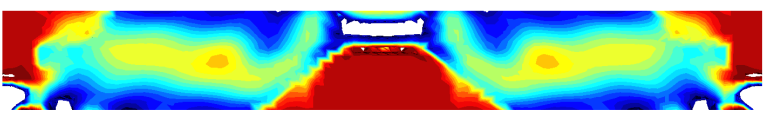

$t_{30}$

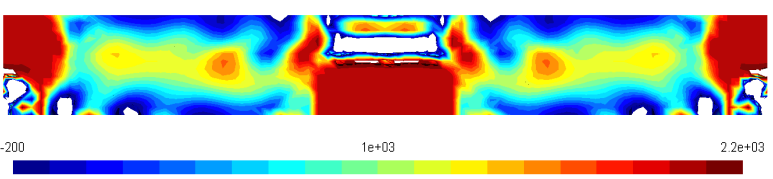

Fig. 21 Evolution of maximum stress eigenvalues in the concrete body (in $\mathrm{kPa}$ )

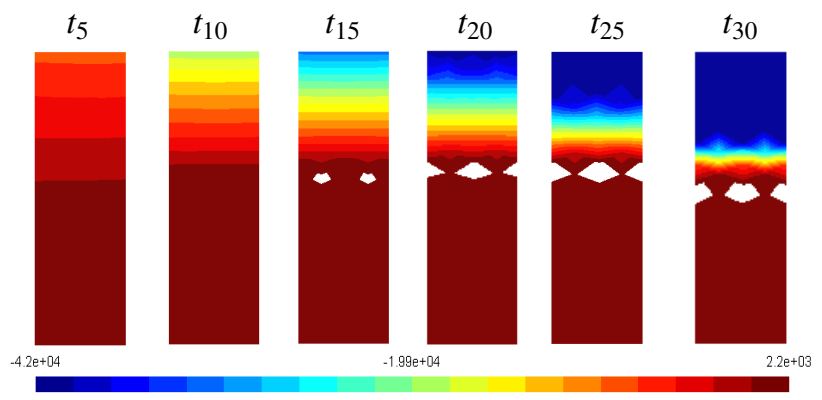

Fig. 22 Evolution of the normal stress $\sigma_{33}(\mathrm{kPa})$ in the mid-span crosssection $\left(x_{3}=5 \mathrm{~m}\right)$

\subsection{Validity of the elastoplastic model}

The main limitation of an elastoplastic approach is the infinite energy it can dissipate after yield stress is reached. Concrete is known to be a brittle material in tension and it cannot sustain high strains. Based on the results presented in the previous section, we suggest a fracture mechanics approach. Considering the traction eigenvalues presented in Figure 21, we identify in Figure 23 the concrete volume $V_{\text {crack }}$ in traction under the load application as the area most likely to suffer from cracking. The energy needed to open a vertical crack in the middle of this area is compared to the energy dissipated in $V_{\text {crack }}$. This fracture mechanism approach has been used for instance in [48] for the study of peeling off in a reinforced concrete beam submitted to three points bending. It presents the advantage to be not dependent on the mesh provided the solution is converged. Indeed, as long as the volume $V_{\text {crack }}$ is 
prescribed and there is no strain localisation, the converged solution is unique.

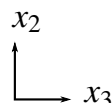

$x_{3}$

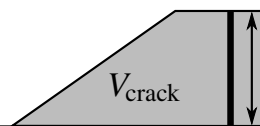

\section{$h$}

Fig. 23 Definition of the potential cracked area

Based on Figure 22, we consider a crack opening of width $b=0.4 \mathrm{~m}$ and height $h=0.8 \mathrm{~m}$ (approximately $60 \%$ of the height of the cross-section). The energy needed to open this crack is thus:

$E_{\text {crack }}=G_{f} \times b \times h$

where $G_{f}$ is the fracture energy. According to the norm CEB-FIP [49], $G_{f}$ is defined as follows:

$G_{f}=G_{f_{0}}\left(\frac{f_{c}}{10}\right)^{0.7}$

$G_{f_{0}}$ is a reference fracture energy based on the size of the aggregates in concrete. Considering an average size of $20 \mathrm{~mm}$, $G_{f_{0}}=36 \mathrm{~N} \cdot \mathrm{m} / \mathrm{m}^{2}$ and Equation (31) yields:

$E_{\text {crack }}=30.4$ N.m

This energy must be compared to the energy dissipated in $V_{\text {crack }}$ defined by:

$E_{\mathrm{diss}}(t)=\int_{0}^{t} \int_{\mathrm{V}_{\text {crack }}} \sigma(\tau): \dot{\boldsymbol{\varepsilon}}^{\mathrm{p}}(\tau) \mathrm{d} V \mathrm{~d} \tau$

The time-discretization of Equation 34 then yields:

$E_{\mathrm{diss}}\left(t_{n}\right)=\sum_{k=1}^{n}\left[\int_{\mathrm{V}_{\text {crack }}} \boldsymbol{\sigma}_{n}: \Delta \boldsymbol{\varepsilon}_{n}^{\mathrm{p}} \mathrm{d} V\right]$.

The computed dissipated energy is presented in Figure 24. As long as concrete is elastic, no energy is dissipated since $\varepsilon^{p}=0$. Once concrete stress reaches $f_{t}$, the energy increases linearly, reflecting a linear evolution of the plastic strain increments (neglecting hardening of concrete, the stress can be considered as constant). The energy drastically increases as soon as plastification in rebars is too important. The energy needed to open the crack is reached at $t_{25}$.

Time step $t_{25}$ corresponding to the crack opening at midspan of the beam has been indicated in Figures 18 and 19. All the time steps subsequent to $t_{25}$ can therefore be considered not valid.

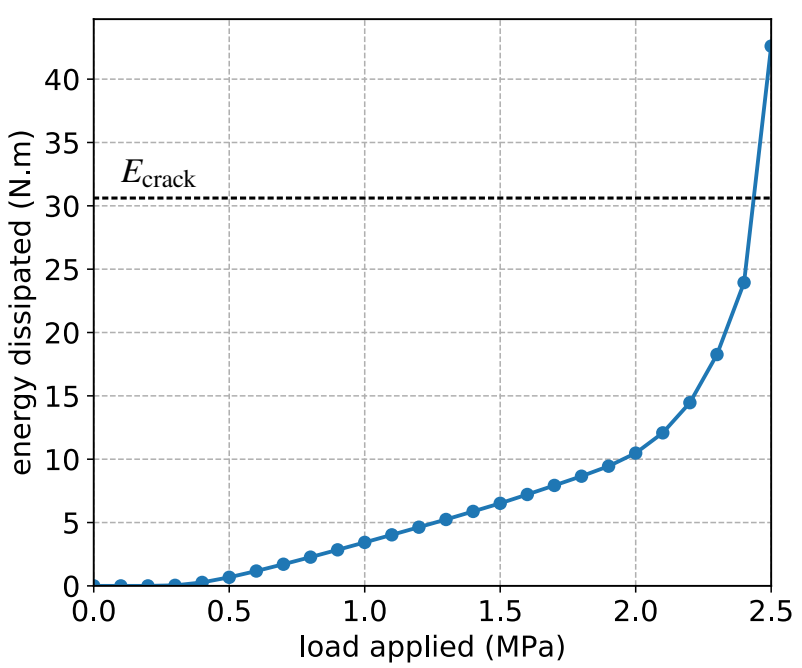

Fig. 24 Energy dissipated in $V_{\text {crack }}$ as function of the load applied

\section{Mesh sensitivity}

\subsection{Case study}

The beam element and rebars have different and independent meshes, as mentioned in Section 4.2. This section is dedicated to the study of its mesh sensitivity. To this purpose, we consider the three points bending case with the I-section presented in Figure 25. This example is taken from the validation document [50] of the documentation of the finite element software Code_Aster.

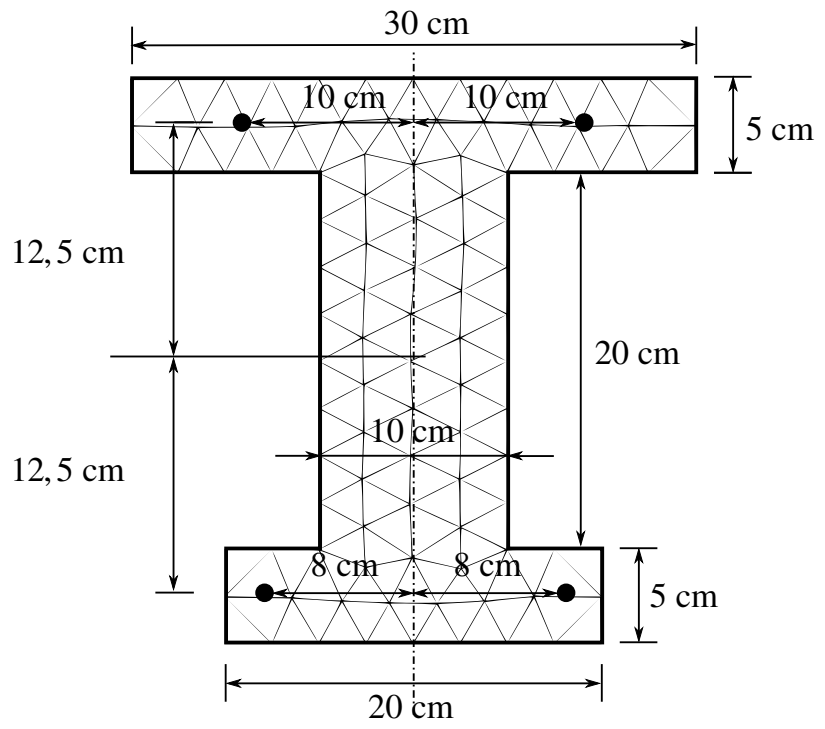

Fig. 25 Geometry and mesh of the I-section

Since we study the mesh sensitivity of the model, the beam is purely elastic. The mechanical properties of the beam are summarized in Table 5. The total sections of the upper 
and lower rebars are respectively $3 \cdot 10^{-4} \mathrm{~m}^{2}$ and $4.10^{-4} \mathrm{~m}^{2}$. The applied load is $F=10 \mathrm{kN}$. No transverse frames are considered here: the beam being elastic, it is free from collapse in shear.

\begin{tabular}{ccc}
\hline & $E$ & $v$ \\
\hline concrete & $21 \mathrm{GPa}$ & 0.2 \\
\hline steel & $210 \mathrm{GPa}$ & - \\
\hline
\end{tabular}

Table 5 Material properties

\subsection{The beam solution}

The kinematics of the beam model is composed of 23 displacement modes. The transverse mesh is fixed and composed of 144 quadratic triangles, as shown in Figure 25.

The longitudinal mesh sensitivity of the beam element is investigated by changing the number of knots defining the interpolation functions mentioned in Section 3.3. The number of knots $n_{\text {knot }}$ takes different values from 2 to 40 . Simultaneously, rebars mesh sensitivity is studied with different discretizations: rebars are successively meshed with $n_{\text {node }}=3$ to $n_{\text {node }}=15$.

\subsection{Results}

The data observed is the maximum vertical displacement $u_{\mathrm{m}}$ at mid-span of the beam. Results are presented in Figure 26 where the Euler solution is also represented and corresponds to the following value of $u_{\mathrm{m}}^{\text {Euler} \text { : }}$

$u_{\mathrm{m}}^{\text {Euler }}=\frac{F L^{3}}{48(E I)^{\mathrm{tot}}}$,

where $(E I)^{\text {tot }}=E I+E^{\text {st }} I^{\text {st }}$. The numerical value is $u_{\mathrm{m}}^{\text {Euler }}=$ $2.273510^{-3} \mathrm{~m}$. Because of the enriched kinematics of the AELD beam model, it is less stiff than the Euler solution.

The more numerous the knots are, the less stiff the solution is. Thus, for a given value of $n_{\text {node }}$, the values of $u_{\mathrm{m}}$ monotonically increase from $n_{\text {knot }}=5$ to $n_{\text {knot }}=40$. On the opposite, the more numerous the nodes are, the stiffer the element is. Thus, for a given value of $n_{\text {knot }}$, each curve monotonically decreases from $n_{\text {node }}=3$ to $n_{\text {node }}=15$. The increase of the number of nodes in each rebar increases the connectivity between the degrees of freedom of the beam element, and consequently makes it stiffer.

Considering the value $u_{\mathrm{m}}\left(n_{\mathrm{knot}}=40, n_{\text {node }}=15\right)$ as the converged value, we define the relative error of each curve to the converged value by:

$e_{1}\left(n_{\mathrm{knot}}\right)=\left|\frac{u_{\mathrm{m}}\left(n_{\mathrm{knot}}, 15\right)-u_{\mathrm{m}}(40,15)}{u_{\mathrm{m}}(40,15)}\right|$

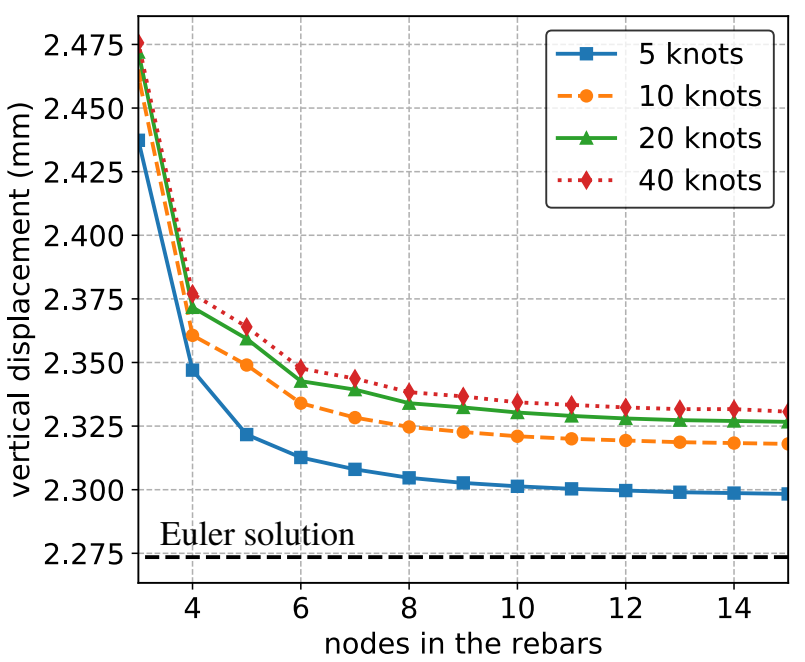

Fig. 26 Maximum vertical displacement for different meshes refinements

\begin{tabular}{cccc}
\hline$n_{\text {knot }}$ & 5 & 10 & 20 \\
\hline$e_{1}$ & $1.3910^{-2}$ & $5.4310^{-3}$ & $1.7210^{-3}$ \\
\hline
\end{tabular}

Table 6 relative error to the converged value $u_{\mathrm{m}}(40,15)$

The values of $e_{1}$ for each curve are presented in Table 6 which shows that the relative error to the converged value is lower than $1 \%$ for $n_{\mathrm{knot}}=10$.

In order to determine the minimum number of nodes in each rebar, the following relative error is defined:

$e_{2}\left(n_{\text {knot }}, n_{\text {node }}\right)=\left|\frac{u_{\mathrm{m}}\left(n_{\text {knot }}, n_{\text {node }}\right)-u_{\mathrm{m}}\left(n_{\text {knot }}, 15\right)}{u_{\mathrm{m}}\left(n_{\mathrm{knot}}, 15\right)}\right|$

where $u_{\mathrm{m}}\left(n_{\mathrm{knot}}, 15\right)$ is considered as the reference for a given value of $n_{\text {knot }}$. The results are gathered in Table 7 .

The number of nodes corresponding to the first value of $e_{2}$ lower than $1 \%$ is $n_{\text {node }}=6$ for each curve. For the present study it can therefore be considered as a sufficient discretization of rebars. This reference case study for mesh sensitivity is a good predictor for the refinement to adopt, even though a similar study should be carried out in plasticity to be completely exhaustive.

\subsection{Computational time}

In terms of computational time, a full computation as the ones presented in this paper takes about 10 minutes. But it worth noticing that the program is not fully optimized yet and that it could therefore still be significantly improved. The computation of the modes is very fast: it never exceeds a few seconds even when the model reaches 30 modes. The two most time-consuming parts of the program are the computation of the plastic strain on each integration point of 


\begin{tabular}{ccccccc}
\hline$n_{\text {node }}$ & 4 & 6 & 8 & 10 & 12 & 14 \\
\hline$e_{2}\left(5, n_{\text {node }}\right)$ & $2.1210^{-2}$ & $6.2410^{-3}$ & $2.7610^{-3}$ & $1.3110^{-3}$ & $5.8010^{-4}$ & $1.4510^{-4}$ \\
\hline$e_{2}\left(10, n_{\text {node }}\right)$ & $1.8410^{-2}$ & $6.9010^{-3}$ & $2.8810^{-3}$ & $1.2910^{-3}$ & $5.7510^{-4}$ & $1.4410^{-4}$ \\
\hline$e_{2}\left(20, n_{\text {node }}\right)$ & $1.9310^{-2}$ & $6.8810^{-3}$ & $3.1510^{-3}$ & $1.5810^{-3}$ & $5.7310^{-4}$ & $1.4310^{-4}$ \\
\hline$e_{2}\left(40, n_{\text {node }}\right)$ & $1.9910^{-2}$ & $7.2910^{-3}$ & $3.2910^{-3}$ & $1.5710^{-3}$ & $7.1510^{-4}$ & $4.2910^{-4}$ \\
\hline
\end{tabular}

Table 7 relative error of each curve for its own converged value

every integration section and the assembly of the tangent stiffness matrix. Indeed those two parts involve loops on all the integration sections and every Gauss points of the 2D cross-section. However those two pieces of the program are very suitable for parallel computing which would drastically improve the global computational performance.

The computational performance of the algorithm must be interpreted and compared to a full 3D model by analyzing the total number of degrees of freedom. The total number of degrees of freedom of the model we present is the multiplication of the number of knots of our longitudinal discretization by the number of modes plus the number of nodes of the reinforcement rebars. In the first example presented the number of knots and modes are respectively about 21 and 28 and the number of nodes in the rebars is 320 , giving a total number of about 900 degrees of freedom. The mesh of the full 3D computation used as a reference solution in the paper is based on the characteristic size of the triangles used for the mesh of the 2D cross-section of the beam solutions. It results in a very refined mesh (146067 tetrahedrons). A mesh more equivalent to our beam element could be made of 477 triangles on a 2D cross-section extruded in 80 prisms along the longitudinal axis, thus respecting the longitudinal discretization used by our beam element for the integration of the 3D plastic strains. This represents about 40000 nodes and therefore 120000 degrees of freedom only for concrete.

\section{Conclusion}

This work introduces a new elastoplastic beam model for reinforced concrete. Both concrete and steel are considered as elastoplastic materials, however there is no limitation in the choice of the constitutive behaviour of concrete and more complex models with damage and softening could be considered with some adaptation. Concrete is associated with a Rankine yield criterion and a closed-form projection of trial stresses on the yield surface was introduced. The concrete body is modelled by a kinematically refined elastoplastic higher-order beam model. Steel is associated with a 1D Von-Mises criterion. Rebars are individually modelled by $1 \mathrm{D}$ bar elements fully embedded in the concrete body, which presents the advantage of enabling various layouts of rebars. The model was validated through a step-by-step procedure.

The example considered highlights the ability of the present beam model to represent complex 3D phenomena.
This ability is provided by the enriched kinematics of the higher-order model considered. The actual failure of the beam is estimated by a fracture mechanics approach. Based on the stress distribution in the concrete, the areas more likely to crack are identified. The energy dissipated in these areas are compared to the fracture energy of concrete. Once the fracture energy is reached, the subsequent computations are considered not significant. This method is a post processing and needs a local knowledge of the stress. It could therefore be improved by implementing a systematic detection of the fracture mechanisms.

The present work is a first approach for modelling reinforced concrete with a rather simple constitutive law. The introduction of more complex constitutive law such as damage is under investigation. This will allow comparison with experimental data on reinforced concrete beams.

\section{References}

1. G. Corre, A. Lebée, K. Sab, M.K. Ferradi, X. Cespedes, International Journal for Numerical Methods in Engineering (2018). DOI 10.1002/nme.5926. URL http://doi.wiley.com/10.1002/nme.5926

2. J. Lubliner, J. Oliver, S. Oller, E. Oñate, International Journal of Solids and Structures 25, 299 (1988)

3. S. Govindjee, G.J. Kay, J.C. Simo, International Journal for Numerical Methods in Engineering 38(21), 3611 (1995). DOI 10.1002/nme.1620382105. URL http://dx.doi.org/10.1002/nme.1620382105

4. J. Mazars, Engineering Fracture Me$\begin{array}{llll}\text { chanics 25(5), } 729 & \text { (1986). DOI }\end{array}$ https://doi.org/10.1016/0013-7944(86)90036-6. URL http://www.sciencedirect.com/science/article/pii/0013794486900366

5. S. Oller, A.H. Barbat, Computational Methods Applied to Mechanical Engineering (2005)

6. W. Chen, Plasticity in Reinforced Concrete. J. Ross Publishing Classics (J. Ross Pub., 2007). URL https://books.google.fr/books?id=FGp-U4hNjggC

7. P.H. Feenstra, R.D. Borst, International Journal of Solids and Structures 33(5), 707 (1996). DOI https://doi.org/10.1016/0020-7683(95)00060-N. URL http://www.sciencedirect.com/science/article/pii/002076839500060N

8. P. Grassl, K. Lundgren, K. Gylltoft, International Journal of Solids and Structures 39(20), 5205 (2002). DOI 
https://doi.org/10.1016/S0020-7683(02)00408-0. URL 26. E. Spacone, S. Limkatanyu, ACI Structural Journal http://www.sciencedirect.com/science/article/pii/S00207683020(21010)

9. M.R. Salari, S. Saeb, K.J. Willam, S.J. Patchet, R.C. Carrasco, Computer Methods in Applied Mechanics and Engineering 193(27), 2625 (2004)

10. P. Grassl, M. Jirásek, International Journal of Solids and Structures 43(22), 7166 (2006). DOI https://doi.org/10.1016/j.ijsolstr.2006.06.032. URL

27. S. Moulin, Elément de poutre multifibre (droite), R3.08.08. Code_Aster (2010)

28. J. Mazars, P. Kotronis, F. Ragueneau, G. Casaux, Computational Mechanics Applied to Mechanical Engineering 195, 7264 (2006) http://www.sciencedirect.com/science/article/pii/S002076830608Qt4deXures (2014)

11. G. Meschke, R. Lackner, A. Mang, International Journal for Numerical Methods in Engineering 42, 703 (1998)

12. ICAB, Calcul des structures en béton (2005)

13. ACI, Building Code Requirements for Structural Concrete and Commentary (2014)

14. J. Dallot, K. Sab, G. Forêt, European Journal of Mechanics - A/Solids 28(1), 166 (2009). DOI 10.1016/j.euromechsol.2008.04.001. URL

30. A. Marini, E. Spacone, ACI Structural Journal 103, 645 (2006)

31. I. Bitar, P. Kotronis, N. Benkemoun, S. Grange, Finite Elements in Analysis \& Design 150(April), 34 (2018). DOI 10.1016/j.finel.2018.07.002. URL https://doi.org/10.1016/j.finel.2018.07.002

32. I. Bitar, S. Grange, P. Kotronis, N. Benkemoun, European Journal of Environmental http://linkinghub.elsevier.com/retrieve/pii/S0997753808000405 and Civil Engineering 22(4), $464 \quad$ (2018).

15. P. de Buhan, B. Sudret, European Journal of Mechanics - A/Solids 18(6), 995 (1999). DOI 10.1016/S0997-7538(99)00109-6. URL DOI 10.1080/19648189.2016.1210031. URL http://dx.doi.org/10.1080/19648189.2016.1210031

16. J. Bleyer, P. de Buhan, European Journal of Mechanics - A/Solids 59, 178 (2016). DOI 10.1016/j.euromechsol.2016.03.002. URL http://dx.doi.org/10.1016/j.euromechsol.2016.03.002

33. J. Navarro Gregori, P. Miguel Sosa, M.A. Fernández http://linkinghub.elsevier.com/retrieve/pii/S0997753816300201 http://ascelibrary.org/doi/10.1061/\%28ASCE\%29ST.1943-

17. M. Epstein, Acta Mechanica 33(3), 229 (1979). DOI 10.1007/BF01175918

18. N. Rizzi, A. Tatone, Journal of Applied Mechanics 63(2), 399 (1996). DOI 10.1115/1.2788878. URL http://appliedmechanics.asmedigitalcollection.asme.org/

19. E. Lofrano, A. Paolone, G. Ruta, Mechanics Research Communications 48, 76 (2013). DOI 10.1016/j.mechrescom.2012.12.008. URL http://dx.doi.org/10.1016/j.mechrescom.2012.12.008

20. G. Piana, E. Lofrano, A. Manuello, G. Ruta, A. Carpinteri, Engineering Structures 135, 246 (2017). DOI 10.1016/j.engstruct.2016.12.038. URL http://dx.doi.org/10.1016/j.engstruct.2016.12.038

21. A. Ibrahimbegović, F. Frey, Int. J. Numer. Methods Eng. 36(2), 303 (1993). DOI 10.1002/nme.1620360209. URL http://dx.doi.org/10.1002/nme.1620360209 http://doi.wiley.com/10.1002/nme.1620360209

22. B.H. Pham, D. Brancherie, L. Davenne, A. Ibrahimbegovic, Computational Mechanics (2012)

23. N.N. Bui, M. Ngo, M. Nikolic, D. Brancherie, A. Ibrahimbegovic, Computers and Structures (2014)

24. D.R. Owen, E. Hinton, Finite elements in plasticity, vol. 271 (Swansea: Pineridge Press, 1980)

25. E. Spacone, F.C. Filippou, F.F. Taucer, Earthquake Engineering and Structural Dynamics 25, 711 (1996)

35. S. Capdevielle, S. Grange, F. Dufour, C. Desprez, European Journal of Environmental and Civil Engineering 20(8), 914 (2016). 3404 (2007). DOI 10.1016/j.engstruct.2007.09.001

34. P. Di Re, D. Addessi, F.C. Filippou, Journal of Structural Engineering 144(6), 04018064 (2018). DOI 10.1061/(ASCE)ST.1943-541X.0002039. URL 541X.0002039

.aBQPartibDe1@80A112Q48189.2015.1084384. URL http://dx.doi.org/10.1080/19648189.2015.1084384

36. J.M. Bairan Garcia, A.R. Mari Bernat, Engineering Structures 29(8), $1722 \quad$ (2007). DOI 10.1016/j.engstruct.2006.09.007. URL http://linkinghub.elsevier.com/retrieve/pii/S0141029606003804

37. J.M. Bairan Garcia, A.R. Mari Bernat, Archives of Computational Methods in Engineering 14(3), 249 (2007). DOI $10.1007 / \mathrm{s} 11831-007-9007-5$. URL http://link.springer.com/10.1007/s11831-007-9007-5

38. S. Mohr, J.M. Bairan Garcia, A.R. Mari Bernat, Engineering Structures 32(12), 3936 (2010). DOI 10.1016/j.engstruct.2010.09.005. URL http://dx.doi.org/10.1016/j.engstruct.2010.09.005

39. M.K. Ferradi, A. Lebée, A. Fliscounakis, X. Cespedes, K. Sab, Comput. Struct. 172, 11 (2016). DOI 10.1016/j.compstruc.2016.05.013. URL http://linkinghub.elsevier.com/retrieve/pii/S0045794916302656

40. G. Corre, A. Lebée, K. Sab, M.K. Ferradi, X. Cespedes, ZAMM - Journal of Applied Mathematics and Mechanics / Zeitschrift für Angewandte Mathematik und Mechanik (2018). DOI 10.1002/zamm.201700180. 
URL http://doi.wiley.com/10.1002/zamm.201700180

41. H. Kupfer, H.K. Hilsdorf, H. Rusch, ACI Journal Proceedings 66, 656 (1969)

42. J. Simo, Comput. Methods Appl. Mech. Eng. 49(1), 55 (1985). DOI 10.1016/0045-7825(85)90050-7. URL http://linkinghub.elsevier.com/retrieve/pii/0045782585900507

43. M. Godio, I. Stefanou, K. Sab, J. Sulem, International Journal for Numerical Methods in Engineering (2016)

44. J. Simo, T. Hughes, Computational Inelasticity. Interdisciplinary applied mathematics (Springer, 1998)

45. M. Silhavy, The Mechanics and Thermodynamics of Continuous Media. Theoretical and Mathematical Physics (Springer Berlin Heidelberg, 2013)

46. STRAINS, (2016)

47. T. De Soza, Eléments MEMBRANE et GRILLE_MEMBRANE, R3.08.07. Code_Aster (2015)

48. S. Radfar, G. Forêt, K. Sab, Revue des composites et des matériaux avancés pp. n², 171-185 (2012). URL https://hal-enpc.archives-ouvertes.fr/hal-00939990

49. Comité Euro International du Béton, CEB-FIP model code 90. CEB Bulletins (1993)

50. J. Flejou, Réponse statique d'une poutre béton armé (section en T) à comportement linéaire, V3.01.111. Code_Aster (2011) 